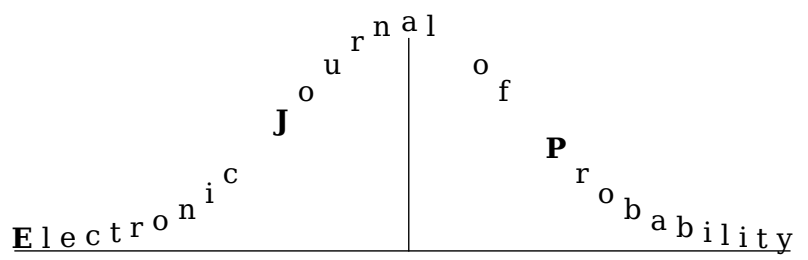

Electron. J. Probab. 26 (2021), article no. 69, 1-27.

ISSN: 1083-6489 https://doi.org/10.1214/21-EJP612

\title{
On the limiting law of the length of the longest common and increasing subsequences in random words with arbitrary distribution*
}

\author{
Clément Deslandes ${ }^{\dagger} \quad$ Christian Houdré ${ }^{\ddagger}$
}

\begin{abstract}
Let $\left(X_{k}\right)_{k>1}$ and $\left(Y_{k}\right)_{k>1}$ be two independent sequences of i.i.d. random variables, with values in a finite and totally ordered alphabet $\mathcal{A}_{m}:=\{1, \ldots, m\}, m \geq 2$, having respective probability mass function $p_{1}^{X}, \ldots, p_{m}^{X}$ and $p_{1}^{Y}, \ldots, p_{m}^{Y}$. Let $L C I_{n}$ be the length of the longest common and weakly increasing subsequences in $X_{1}, \ldots, X_{n}$ and $Y_{1}, \ldots, Y_{n}$. Once properly centered and normalized, $L C I_{n}$ is shown to have a limiting distribution which is expressed as a functional of two independent multidimensional Brownian motions.
\end{abstract}

Keywords: random words; longest common subsequences; longest increasing subsequences; weak convergence; optimal alignment; last passage percolation; random matrices. MSC2020 subject classifications: 05A05; 60C05; 60F05.

Submitted to EJP on September 3, 2019, final version accepted on March 29, 2021.

Supersedes arXiv: 1906.06544.

\section{Introduction and preliminary results}

\subsection{Introduction}

We analyze the asymptotic behavior of $L C I_{n}$, the length of the longest common subsequences in random words with an additional weakly increasing requirement. Throughout, $\left(X_{k}\right)_{k \geq 1}$ and $\left(Y_{k}\right)_{k \geq 1}$ are two independent sequences of i.i.d. random variables with values in the finite totally ordered alphabet $\mathcal{A}_{m}:=\{1, \ldots, m\}, m \geq 2$, and respective pmf $p_{1}^{X}, \ldots, p_{m}^{X}, p_{i}^{X}>0, i=1, \ldots, m$ and $p_{1}^{Y}, \ldots, p_{m}^{Y}, p_{i}^{Y}>0, i=1, \ldots, m$. Next, $L C I_{n}$, the length of the longest common and weakly increasing subsequences of the two random words $X_{1} \cdots X_{n}$ and $Y_{1} \cdots Y_{n}$, is the largest integer $r \in\{1, \ldots, n\}$ such that there exist $1 \leq i_{1}<\cdots<i_{r} \leq n$ and $1 \leq j_{1}<\cdots<j_{r} \leq n$ such that

${ }^{*}$ Research supported in part by the grant $\sharp 524678$ from the Simons Foundation.

${ }^{\dagger}$ C.M.A.P. Ecole Polytechnique, Palaiseau, 91120, France \& Georgia Institute of Technology, Atlanta, GA, 30332, USA. E-mail: clement.deslandes@poytechnique.edu

${ }^{\ddagger}$ School of Mathematics, Georgia Institute of Technology, Atlanta, GA, 30332, USA. E-mail: houdre@math. gatech.edu 
On the limiting law of the length of the longest common and increasing subsequences

- $\forall s \in\{1, \ldots, r\}, X_{i_{s}}=Y_{j_{s}}$,

- $X_{i_{1}} \leq X_{i_{2}} \leq \cdots \leq X_{i_{r}}$ and $Y_{j_{1}} \leq Y_{j_{2}} \leq \cdots \leq Y_{j_{r}}$

and if no integer satisfies these two conditions, we set $L C I_{n}=0$.

A thorough discussion of the study of $L C I_{n}$, with potential applications, and a more complete bibliography, is present in [2], where the following is further proved (below, as usual, $\wedge$ is short for minimum):

Theorem 1.1. Let $X_{k}$ and $Y_{k}(k=1,2, \ldots)$ be uniformly distributed over $\{1, \ldots, m\}$. Then,

$$
\begin{array}{r}
\frac{L C I_{n}-n / m}{\sqrt{n / m}} \underset{\overline{n \rightarrow \infty}}{\Longrightarrow} \max _{0=t_{0} \leq t_{1} \leq \cdots \leq t_{m}=1}\left[\left(-\frac{1}{m} \sum_{i=1}^{m} B_{i}^{X}(1)+\sum_{i=1}^{m}\left(B_{i}^{X}\left(t_{i}\right)-B_{i}^{X}\left(t_{i-1}\right)\right)\right) \wedge\right. \\
\left.\left(-\frac{1}{m} \sum_{i=1}^{m} B_{i}^{Y}(1)+\sum_{i=1}^{m}\left(B_{i}^{Y}\left(t_{i}\right)-B_{i}^{Y}\left(t_{i-1}\right)\right)\right)\right],
\end{array}
$$

where $B^{X}$ and $B^{Y}$ are two independent $m$-dimensional standard Brownian motions on $[0,1]$.

The results of [2] extended (and corrected) the proof of the case $m=2$ analyzed in [4] and also conjectured the following generalization:

Theorem 1.2. Let $X_{k}$ and $Y_{k}(k=1,2, \ldots)$ have the same distribution, let $p_{\max }=$ $\max _{i \in\{1, \ldots, m\}} p_{i}^{X}$ and let $k^{*}$ be its multiplicity. Then

$$
\begin{aligned}
& \frac{L C I_{n}-n p_{\max }}{\sqrt{n p_{\max }}} \underset{n \rightarrow \infty}{\Longrightarrow} \max _{0=t_{0} \leq t_{1} \leq \cdots \leq t_{k^{*}}=1}\left[\left(\frac{\sqrt{1-k^{*} p_{\max }}-1}{k^{*}} \sum_{i=1}^{k^{*}} B_{i}^{X}(1)+\right.\right. \\
& \left.\left.\sum_{i=1}^{k^{*}}\left(B_{i}^{X}\left(t_{i}\right)-B_{i}^{X}\left(t_{i-1}\right)\right)\right) \wedge\left(\frac{\sqrt{1-k^{*} p_{\max }}-1}{k^{*}} \sum_{i=1}^{k^{*}} B_{i}^{Y}(1)+\sum_{i=1}^{k^{*}}\left(B_{i}^{Y}\left(t_{i}\right)-B_{i}^{Y}\left(t_{i-1}\right)\right)\right)\right],
\end{aligned}
$$

where $B^{X}$ and $B^{Y}$ are two independent $k^{*}$-dimensional standard Brownian motions on $[0,1]$.

Clearly, in case $k^{*}=m$, the two limiting distributions in (1.1) and (1.2) are the same but they differ otherwise. Indeed, (1.1) involves two independent $m$-dimensional Brownian motions while (1.2) involves $k^{*}$-dimensional ones. So, in particular, if $k^{*}=1$, then the right-hand side of (1.2) is just the minimum of two independent centered normal random variables. In view of the results obtained in the one-sequence case, e.g., see [5], [1], and the many references therein, it is tantalizing to conjecture that both the right-hand side of (1.1) and of (1.2) can be realized as maximal eigenvalues of some Gaussian random matrix models.

Below, we aim to obtain the limiting distribution of $L C I_{n}$, without assuming that the $X_{k}$ and $Y_{k}(k=1,2, \ldots)$ have the same distribution; providing also an alternative proof of Theorem 1.1 as well as a proof of the conjectured (1.2). A brief description of the content of our notes is as follows: the rest of the current section is devoted to studying the asymptotic mean of $L C I_{n}$. This asymptotic mean result is already not so predictable and allows for the proper centering in the limiting theorem whose proof is provided in the next section. The third and final section is mainly devoted to studying extensions and complements, such as results for sequences with blocks and infinite countable alphabets. 
On the limiting law of the length of the longest common and increasing subsequences

\subsection{Probability}

For $i \in\{1, \ldots, m\}$ and $j \in\{1, \ldots, n\}$, let $\ell \in \mathbb{N}=\{0,1,2, \ldots\}$ be such that $j+\ell \leq n+1$, and let

$$
N_{j, \ell}^{X, i}=\sum_{k=0}^{\ell-1} \mathbb{1}_{X_{j+k}=i} \quad\left(\text { resp. } N_{j, \ell}^{Y, i}=\sum_{k=0}^{\ell-1} \mathbb{1}_{Y_{j+k}=i}\right),
$$

be simply the number of letters $i$ between, and including, $j$ and $j+\ell-1$ in $X_{1}, \ldots, X_{n}$ (resp. $Y_{1}, \ldots, Y_{n}$ ), with the convention that the sum is zero in case $\ell=0$. From the very definition of $L C I_{n}$, it is clear that

$$
L C I_{n}=\max _{\substack{\ell^{X}, \ell^{Y} \in \mathbb{N}^{m} \\ \ell_{1}^{X}+\cdots+\ell_{m}^{X}=n\\}}\left(N_{1, \ell_{1}^{X}}^{X, 1} \wedge N_{1, \ell_{1}^{Y}}^{Y, 1}+\cdots+N_{\ell_{1}^{X}, \ell_{2}^{X}}^{X, 2} \wedge N_{\ell_{1}^{Y}, \ell_{2}^{Y}}^{Y, 2}+\cdots+N_{\ell_{1}^{X}+\cdots+\ell_{m-1}^{X}, \ell_{m}^{X}}^{X, m} \wedge N_{\ell_{1}^{Y}+\cdots+\ell_{m-1}^{Y}, \ell_{m}^{Y}}^{Y, m}\right) .
$$

Next, let $\Lambda=\left\{\lambda \in\left(\mathbb{R}_{+}\right)^{m}=[0,+\infty)^{m}: \lambda_{1}+\cdots+\lambda_{m}=1\right\}$. For $\lambda \in \Lambda$, let

$$
\ell^{n}(\lambda)_{i}=\left\lfloor\left(\lambda_{1}+\cdots+\lambda_{i}\right) n\right\rfloor-\left\lfloor\left(\lambda_{1}+\cdots+\lambda_{i-1}\right) n\right\rfloor,
$$

where $\lfloor$.$\rfloor is the usual integer part, aka the floor, function. When \lambda$ runs through $\Lambda$, $\ell^{n}(\lambda)=\left(\ell^{n}(\lambda)_{1}, \ldots, \ell^{n}(\lambda)_{m}\right)$ runs exactly through $\left\{\ell \in \mathbb{N}^{m}: \ell_{1}+\cdots+\ell_{m}=n\right\}$, so

$$
\begin{aligned}
L C I_{n}=\max _{\lambda^{X}, \lambda^{Y} \in \Lambda}\left(N_{1, \ell^{n}\left(\lambda^{X}\right)_{1}}^{X, 1} \wedge N_{1, \ell^{n}\left(\lambda^{Y}\right)_{1}}^{Y, 1}+N_{\ell^{n}\left(\lambda^{X}\right)_{1}, \ell^{n}\left(\lambda^{X}\right)_{2}}^{X, 2} \wedge N_{\ell^{n}\left(\lambda^{Y}\right)_{1}, \ell^{n}\left(\lambda^{Y}\right)_{2}}^{Y, 2}+\ldots\right. \\
\left.+N_{\ell^{n}\left(\lambda^{X}\right)_{1}+\cdots+\ell^{n}\left(\lambda^{X}\right)_{m-1}, \ell^{n}\left(\lambda^{X}\right)_{m}}^{X, m} \wedge N_{\ell^{n}\left(\lambda^{Y}\right)_{1}+\cdots+\ell^{n}\left(\lambda^{Y}\right)_{m-1}, \ell^{n}\left(\lambda^{Y}\right)_{m}}^{Y, m}\right)
\end{aligned}
$$

For ease of notations, throughout the paper, for all $x \in\left(\mathbb{R}^{m}\right)^{2}$, we write $x=\left(x^{X}, x^{Y}\right)$ so, for example, above, $\lambda^{X}, \lambda^{Y} \in \Lambda$ becomes $\lambda \in \Lambda^{2}$.

For $i \in\{1, \ldots, m\}$ and $t \in[0,1]$, let now

$$
\widetilde{B}_{i}^{n, X}(t)=\frac{N_{1,\lfloor t n\rfloor}^{X, i}-p_{i}^{X} t n}{\sqrt{p_{i}^{X}\left(1-p_{i}^{X}\right) n}}, \quad\left(\operatorname{resp} . \widetilde{B}_{i}^{n, Y}(t)=\frac{N_{1,\lfloor t n\rfloor}^{Y, i}-p_{i}^{Y} t n}{\sqrt{p_{i}^{Y}\left(1-p_{i}^{Y}\right) n}}\right),
$$

and for $\lambda \in \Lambda^{2}$, let

$$
\begin{aligned}
& \widetilde{V}_{i}^{n, X}\left(\lambda^{X}\right)=\sqrt{p_{i}^{X}\left(1-p_{i}^{X}\right)}\left(\widetilde{B}_{i}^{n, X}\left(\lambda_{1}^{X}+\cdots+\lambda_{i}^{X}\right)-\widetilde{B}_{i}^{n, X}\left(\lambda_{1}^{X}+\cdots+\lambda_{i-1}^{X}\right)\right), \\
& \widetilde{V}_{i}^{n, Y}\left(\lambda^{Y}\right)=\sqrt{p_{i}^{Y}\left(1-p_{i}^{Y}\right)}\left(\widetilde{B}_{i}^{n, Y}\left(\lambda_{1}^{Y}+\cdots+\lambda_{i}^{Y}\right)-\widetilde{B}_{i}^{n, Y}\left(\lambda_{1}^{Y}+\cdots+\lambda_{i-1}^{Y}\right)\right),
\end{aligned}
$$

so that (1.4) becomes

$$
L C I_{n}=\max _{\lambda \in \Lambda^{2}} \sum_{i=1}^{m}\left[\left(n p_{i}^{X} \lambda_{i}^{X}+\sqrt{n} \widetilde{V}_{i}^{n, X}\left(\lambda^{X}\right)\right) \wedge\left(n p_{i}^{Y} \lambda_{i}^{Y}+\sqrt{n} \widetilde{V}_{i}^{n, Y}\left(\lambda^{Y}\right)\right)\right] .
$$

The above identity provides a representation of $L C I_{n}$ as a maximum over the locations, $\lambda \in \Lambda^{2}$, where to pick in each word $X_{1}, \ldots, X_{n}$ and $Y_{1}, \ldots, Y_{n}$, the letters $1,2, \ldots, m$ in order to form a common sub-word. This is different from the approach in [2], where the maximum is over the numbers of letters $1,2, \ldots, m$ in a common sub-word. Of course the two representations are equivalent. However, the advantage of our approach is that $\lambda$ takes its values in a deterministic set, as opposed to a random set. 
On the limiting law of the length of the longest common and increasing subsequences

In order to keep dealing with maxima it will be convenient to replace $\widetilde{B}_{i}^{n}$ in (1.5) by its continuous alternative: for $i \in\{1, \ldots, m\}$ and $t \in[0,1]$, let

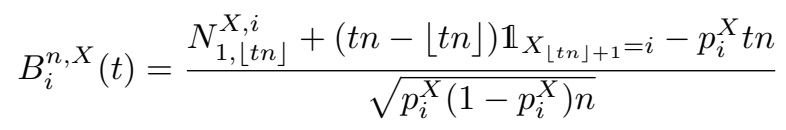

and

$$
B_{i}^{n, Y}(t)=\frac{N_{1,\lfloor t n\rfloor}^{Y, i}+(t n-\lfloor t n\rfloor) \mathbb{1}_{Y_{\lfloor t n\rfloor+1}=i}-p_{i}^{Y} t n}{\sqrt{p_{i}^{Y}\left(1-p_{i}^{Y}\right) n}} .
$$

Next define $V^{n, X}, V^{n, Y}$ just as in (1.6) and (1.7), replacing $\widetilde{B}$ by $B$, and let

$$
L C I_{n}^{c}=\max _{\lambda \in \Lambda^{2}} \sum_{i=1}^{m}\left[\left(n p_{i}^{X} \lambda_{i}^{X}+\sqrt{n} V_{i}^{n, X}(\lambda)\right) \wedge\left(n p_{i}^{Y} \lambda^{Y}+\sqrt{n} V_{i}^{n, Y}(\lambda)\right)\right] .
$$

Our analysis rests upon estimating the variations of $B_{i}^{n, X}$ and of $B_{i}^{n, Y}$. To do so, let $\eta \in(0,1 / 6)$ and let $A_{n}^{\eta}$ be the event:

$$
\begin{gathered}
\forall i \in\{1, \ldots, m\}, \forall j \in\{1, \ldots, n\}, \forall \ell \in\{0, \ldots, n+1-j\},\left|\frac{N_{j, \ell}^{X, i}-p_{i}^{X} \ell}{\sqrt{n}}\right| \leq \frac{n^{\eta}}{2} \sqrt{\frac{\ell}{n}}, \\
\text { and }\left|\frac{N_{j, \ell}^{Y, i}-p_{i}^{Y} \ell}{\sqrt{n}}\right| \leq \frac{n^{\eta}}{2} \sqrt{\frac{\ell}{n}} .
\end{gathered}
$$

By Hoeffding's inequality,

$$
1-\mathbb{P}\left(A_{n}^{\eta}\right) \leq 2 n(n+1) m \exp \left(-\frac{n^{2 \eta}}{2}\right),
$$

and so if $A_{n}^{\eta}$ occurs, then for all $x, y$ in $[0,1]$ and $i \in\{1, \ldots, m\}$,

$$
\left|\sqrt{p_{i}^{X}\left(1-p_{i}^{X}\right)}\left(B_{i}^{n, X}(y)-B_{i}^{n, X}(x)\right)\right| \leq \frac{n^{\eta}}{2} \sqrt{|y-x|+\frac{1}{n}} .
$$

and in particular,

$$
\left|\sqrt{p_{i}^{X}\left(1-p_{i}^{X}\right)}\left(B_{i}^{n, X}(y)-B_{i}^{n, X}(x)\right)\right| \leq \frac{n^{\eta}}{2} \sqrt{|y-x|}+\frac{n^{\eta-1 / 2}}{2} \leq n^{\eta},
$$

and the same applies to $Y$ instead of $X$.

\subsection{Asymptotic mean: distinct cases}

Let us investigate the limiting behavior of $L C I_{n} / n$. From (1.8),

$$
\frac{L C I_{n}}{n}=\max _{\lambda \in \Lambda^{2}} \sum_{i=1}^{m}\left[\left(p_{i}^{X} \lambda_{i}^{X}+\frac{\widetilde{V}_{i}^{n, X}\left(\lambda^{X}\right)}{\sqrt{n}}\right) \wedge\left(p_{i}^{Y} \lambda_{i}^{Y}+\frac{\widetilde{V}_{i}^{n, Y}\left(\lambda^{Y}\right)}{\sqrt{n}}\right)\right] .
$$

Note that $\left|\widetilde{V}_{i}^{n, X}\left(\lambda^{X}\right)-V_{i}^{n, X}\left(\lambda^{X}\right)\right| \leq 1 / \sqrt{n}$ (and similarly for $Y$ ). Thus, using (throughout the paper) the following elementary inequality, valid for any $a, b, c, d \in \mathbb{R}$,

$$
|a \wedge b-(a+c) \wedge(b+d)| \leq \max (|c|,|d|),
$$

we get

$$
\left|\frac{L C I_{n}}{n}-\frac{L C I_{n}^{c}}{n}\right| \leq \frac{m}{n} .
$$


On the limiting law of the length of the longest common and increasing subsequences

Moreover, if $A_{n}^{\eta}$ occurs, then for all $\lambda \in \Lambda^{2}$,

$$
\left|\sum_{i=1}^{m}\left[\left(p_{i}^{X} \lambda_{i}^{X}+\frac{V_{i}^{n, X}\left(\lambda^{X}\right)}{\sqrt{n}}\right) \wedge\left(p_{i}^{Y} \lambda_{i}^{Y}+\frac{V_{i}^{n, Y}\left(\lambda^{Y}\right)}{\sqrt{n}}\right)\right]-\sum_{i=1}^{m}\left[\left(p_{i}^{X} \lambda_{i}^{X}\right) \wedge\left(p_{i}^{Y} \lambda_{i}^{Y}\right)\right]\right| \leq \frac{m}{n^{1 / 2-\eta}},
$$

so, letting $f:\left(\mathbb{R}^{m}\right)^{2} \rightarrow \mathbb{R}$ be given via

$$
f:\left(y^{X}, y^{Y}\right) \mapsto \sum_{i=1}^{m}\left[\left(p_{i}^{X} y_{i}^{X}\right) \wedge\left(p_{i}^{Y} y_{i}^{Y}\right)\right]
$$

we have:

$$
\left|\frac{L C I_{n}}{n}-\max _{\lambda \in \Lambda^{2}} f(\lambda)\right| \leq m n^{\eta-1 / 2} .
$$

By the Borel-Cantelli lemma (recalling (1.9)), almost surely, eventually $A_{n}^{\eta}$ occurs so $L C I_{n}^{c} / n$ and $L C I_{n} / n$ both converge almost surely to

$$
e_{\max }:=\max _{\lambda \in \Lambda^{2}} f(\lambda) \text {. }
$$

From

we also get by dominated convergence

$$
\frac{L C I_{n}}{n} \underset{n \rightarrow \infty}{\longrightarrow} e_{\max }, \text { a.s., }
$$

$$
\frac{\mathbb{E} L C I_{n}}{n} \underset{n \rightarrow \infty}{\longrightarrow} e_{\max }
$$

One can think of $e_{\max }$ as the length ratio of the longest common and increasing subsequences in a continuous, non-probabilistic setup: the letters have density masses $p_{1}^{X}, p_{2}^{X}, \ldots, p_{m}^{X}$ and $p_{1}^{Y}, p_{2}^{Y}, \ldots, p_{m}^{Y}$.

Now, let

$$
U=\left\{u \in\left(\mathbb{R}_{+}\right)^{m}: \frac{u_{1}}{p_{1}^{X}}+\cdots+\frac{u_{m}}{p_{m}^{X}} \leq 1, \frac{u_{1}}{p_{1}^{Y}}+\cdots+\frac{u_{m}}{p_{m}^{Y}} \leq 1\right\},
$$

and let $\phi: \mathbb{R}^{m} \rightarrow \mathbb{R}$ be given by $\phi: u \mapsto u_{1}+\cdots+u_{m}$.

On $U$, there is a correspondence between $f$ in (1.12), and the above $\phi$. Indeed, for $\lambda \in \Lambda^{2}$, defining $u$ by $u_{i}=\left(p_{i}^{X} \lambda_{i}^{X}\right) \wedge\left(p_{i}^{Y} \lambda_{i}^{Y}\right), f(\lambda)=\phi(u)$, and for $u \in U$, there exists $\lambda \in \Lambda^{2}$, such that $\lambda_{i}^{X} \geq u_{i} / p_{i}^{X}$ and $\lambda_{i}^{Y} \geq u_{i} / p_{i}^{Y}$ so that $f(\lambda) \geq \phi(u)$. Therefore, $e_{\max }=\max _{u \in U} \phi(u)$. Also, let

$$
K_{\Lambda^{2}}=f^{-1}\left(\left\{e_{\max }\right\}\right) \cap \Lambda^{2} \text {, and } L_{U}=\phi^{-1}\left(\left\{e_{\max }\right\}\right) \cap U .
$$

The above correspondence provides for each element of $K_{\Lambda^{2}}$ an element of $L_{U}$, and for each element of $L_{U}$ at least one element of $K_{\Lambda^{2}}$ (if one of the two inequalities defining $U$ is strict, then there is more than one way to define the corresponding $\lambda$ ). Next, let $I$ be the set of integers $i \in\{1, \ldots, m\}$ such that there exists $u^{i} \in L_{U}$ with $u_{i}^{i}>0$. One can think of $I$ as the letters that can be used to maximize $\phi$, or, equivalently, to maximize $f$. Let

$$
u^{I}=\frac{1}{|I|} \sum_{i \in I} u^{i},
$$

so $u^{I} \in L_{U}$ and for all $i \in I, u_{i}^{I}>0$. Thanks to the above correspondence, we define (and will use throughout the paper) $a \in \Lambda^{2}$ such that $a_{i}^{X}=a_{i}^{Y}=0$ for all $i \notin I$ and $a_{i}^{X} \geq u_{i}^{I} / p_{i}^{X}$, $a_{i}^{Y} \geq u_{i}^{I} / p_{i}^{Y}$, for all $i \in I$ ( $a$ is a correspondent of $u_{I}$ ). Since $f(a) \geq \phi\left(u^{I}\right)=e_{\max }, a \in K_{\Lambda^{2}}$. We shall see, and use, that when restricting the alphabet to $I$, asymptotically (when properly centered and normalized) the distribution of $L C I_{n}$ remains unchanged.

Two distinct cases need to be analyzed in order to study the limiting distribution of $L C I_{n}$. 
On the limiting law of the length of the longest common and increasing subsequences

Case a) There exists $u \in L_{U}$ such that $\frac{u_{1}}{p_{1}^{X}}+\cdots+\frac{u_{m}}{p_{m}^{X}}=1$ and $\frac{u_{1}}{p_{1}^{Y}}+\cdots+\frac{u_{m}}{p_{m}^{Y}}<1$.

For example, when $p^{X}=(3 / 8,3 / 8,1 / 4)$ and $p^{Y}=(1 / 2,3 / 8,1 / 8)$. Here the maximum is $3 / 8$, and $I=\{1,2\}$.

Heuristically, this case indicates that the length of the common words is limited by the word $X_{1} \cdots X_{n}$ and not by $Y_{1} \cdots Y_{n}$. Using the correspondence between $L_{U}$ and $K_{\Lambda^{2}}$, this case is equivalent to the following statement: there exists $\lambda \in K_{\Lambda^{2}}$ such that for all $i \in\{1, \ldots, m\}, p_{i}^{X} \lambda_{i}^{X} \leq p_{i}^{Y} \lambda_{i}^{Y}$ with at least one strict inequality. In this case, one has:

Lemma 1.3. Let $p_{\max }^{X}=\max _{i \in\{1, \ldots, m\}} p_{i}^{X}$. Then $I=\left\{i \in\{1, \ldots, m\}: p_{i}^{X}=p_{\max }^{X}\right\}$ and $e_{\max }=p_{\max }^{X}$. Moreover there exists $i_{1} \in I$ such that $p_{i_{1}}^{Y}>p_{\max }^{X}$.

Proof. Let $i, j \in\{1, \ldots, m\}$ be such that $p_{i}^{X}<p_{j}^{X}$, and assume, by contradiction, that $i \in I$. Let $u \in L_{U}$ satisfying $\frac{u_{1}}{p_{1}^{X}}+\cdots+\frac{u_{m}}{p_{m}^{X}}=1$ and $\frac{u_{1}}{p_{1}^{Y}}+\cdots+\frac{u_{m}}{p_{m}^{Y}}<1$, and let $v=\left(u^{i}+u\right) / 2$, so that $v \in U, v_{i}>0, \frac{v_{1}}{p_{1}^{X}}+\cdots+\frac{v_{m}}{p_{m}^{X}} \leq 1$ and $\frac{v_{1}}{p_{1}^{Y}}+\cdots+\frac{v_{m}}{p_{m}^{Y}}<1$. Let, for $\varepsilon>0, v(\varepsilon)$ be the vector $v$ except at the coordinates $i$ and $j$ where $v(\varepsilon)_{i}:=v_{i}-\varepsilon p_{i}^{X}$ and $v(\varepsilon)_{j}:=v_{j}+\varepsilon p_{j}^{X}$. It is clear that, when $\varepsilon$ is small enough, $v(\varepsilon) \in U$ and $\phi(v(\varepsilon))=e_{\max }+\varepsilon\left(p_{j}^{X}-p_{i}^{X}\right)>e_{\max }$, leading to a contradiction. Hence $I \subset\left\{i \in\{1, \ldots, m\}: p_{i}^{X}=p_{\max }^{X}\right\}$. Reciprocally, let $i \in\{1, \ldots, m\}$ be such that $p_{i}^{X}=p_{\max }^{X}$ and let $j \in I$. If $i=j$ we are done. Otherwise, one can slightly change $u$ by adding $\varepsilon$ to the $i$ th coordinate and subtracting $\varepsilon$ to the $j$ th coordinate so that $\phi(u)$ remains unchanged, and $u$ is still in $U$ (for $\varepsilon$ small enough), so $I=\left\{i \in\{1, \ldots, m\}: p_{i}^{X}=p_{\max }^{X}\right\}$.

Since $\frac{u_{1}}{p_{1}^{X}}+\cdots+\frac{u_{m}}{p_{m}^{X}}=\sum_{i \in I} \frac{u_{i}}{p_{\max }^{X}}>\sum_{i \in I} \frac{u_{i}}{p_{i}^{Y}}$, there exists $i_{1} \in I$ such that $p_{i_{1}}^{Y}>p_{\max }^{X}$. It is finally clear that $e_{\max }=p_{\max }^{X}$, completing the proof.

As a consequence of the above lemma, we prove next that

$$
J:=\left\{\lambda^{X} \in \Lambda: \forall i \notin I, \lambda_{i}^{X}=0, \sum_{i \in I} \frac{\lambda_{i}^{X}}{p_{i}^{Y}} \leq \frac{1}{p_{\max }^{X}}\right\}=\left\{\lambda^{X}: \lambda \in K_{\Lambda^{2}}\right\},
$$

(in particular, this set is non-empty which is all that is really needed in the rest of the proof). To show this equality, first note that $\left\{\lambda^{X}: \lambda \in K_{\Lambda^{2}}\right\} \subset J$ since, indeed, when $\lambda \in K_{\Lambda^{2}}$, for every $i \in I, p_{\max }^{X} \lambda_{i}^{X} \leq p_{i}^{Y} \lambda_{i}^{Y}$ and then take the sum. Conversely, if $\lambda^{X} \in J$, $\sum_{i \in I} p_{\max }^{X} \lambda_{i}^{X} / p_{i}^{Y} \leq 1$, so let $\lambda^{Y}$ be such that for every $i \in I, \lambda_{i}^{Y} \geq p_{\max }^{X} \lambda_{i}^{X} / p_{i}^{Y}$ and $\sum_{i \in I} \lambda_{i}^{Y}=1$, while for $i \in I^{c}$, let $\lambda_{i}^{Y}=0$. Clearly, $\lambda \in K_{\Lambda^{2}}$.

Case b) For all $u \in L_{U}, \frac{u_{1}}{p_{1}^{X}}+\cdots+\frac{u_{m}}{p_{m}^{X}}=\frac{u_{1}}{p_{1}^{Y}}+\cdots+\frac{u_{m}}{p_{m}^{Y}}=1$.

Heuristically, this second case indicates that in order to form the longest common words, it is necessary to make full use of both words. Using the correspondence between $L_{U}$ and $K_{\Lambda^{2}}$, this case is equivalent to the following: for all $\lambda \in K_{\Lambda^{2}}$, for all $i \in\{1, \ldots, m\}, p_{i}^{X} \lambda_{i}^{X}=p_{i}^{Y} \lambda_{i}^{Y}$. We can further distinguish two subcases, namely, we are in Case b1) if each coordinate of $P^{X}:=\left(1 / p_{i}^{X}\right)_{i \in I} \in \mathbb{R}^{I}$ is equal to each coordinate of $P^{Y}=\left(1 / p_{i}^{Y}\right)_{i \in I} \in \mathbb{R}^{I}$, and in Case b2) otherwise.

For example, if $p^{X}=(1 / 3,1 / 3,2 / 9,1 / 9)$ and $p^{Y}=(1 / 3,1 / 3,1 / 9,2 / 9)$, we are in Case b1) and $e_{\max }=1 / 3$. If $p^{X}=(2 / 3,1 / 6,1 / 6)$ and $p^{Y}=(1 / 6,2 / 3,1 / 6)$, we are in Case b2) and $e_{\max }=4 / 15$. In both of these examples, $I=\{1,2\}$.

Below $\operatorname{Span}\left(P^{X}\right)\left(\right.$ resp. $\left.\operatorname{Span}\left(P^{Y}\right)\right)$ is the linear span of $P^{X}$ (resp. $\left.P^{Y}\right)$.

Lemma 1.4. In Case b2), there exists a unique pair of reals $s, t$ such that $s P^{X}+t P^{Y}=$ $(1)_{i \in I}$

Proof. The only alternatives to Case b1) are: $P^{X}$ and $P^{Y}$ are linearly independent, or $P^{X}$ and $P^{Y}$ are linearly dependent and $P^{X} \neq P^{Y}$. If the latter, given that $P^{X}$ and $P^{Y}$ have positive coordinates, $P^{X}<P^{Y}$ (coordinate by coordinate) or $P^{Y}<P^{X}$. But 
On the limiting law of the length of the longest common and increasing subsequences

$P^{X}<P^{Y}$ clearly implies that Case a) occurs, and not Case b) leading to a contradiction (and similarly $P^{Y}<P^{X}$ ). Therefore, the only alternative to Case b1) is for $P^{X}$ and $P^{Y}$ to be linearly independent. We now prove that $H:=(1)_{i \in I} \in \operatorname{Span}\left(P^{X}, P^{Y}\right)$. To do so, we use an elementary duality result: if $E$ is a finite-dimensional space with dual $E^{*}$, and if $l_{1}, l_{2}, l_{3} \in E^{*}$, then $\operatorname{Ker}\left(l_{1}\right) \cap \operatorname{Ker}\left(l_{2}\right) \subset \operatorname{Ker}\left(l_{3}\right)$ if and only if $l_{3} \in \operatorname{Span}\left(l_{1}, l_{2}\right)$. Indeed, considering the restrictions $l_{2 \mid \operatorname{Ker}\left(l_{1}\right)}$ and $l_{3 \mid \operatorname{Ker}\left(l_{1}\right)}$ of $l_{2}$ and $l_{3}$ to the subspace $\operatorname{Ker}\left(l_{1}\right)$, we have $\operatorname{Ker}\left(l_{2 \mid \operatorname{Ker}\left(l_{1}\right)}\right) \subset \operatorname{Ker}\left(l_{3 \mid \operatorname{Ker}\left(l_{1}\right)}\right)$. Therefore, $l_{3 \mid \operatorname{Ker}\left(l_{1}\right)}=\lambda l_{2 \mid \operatorname{Ker}\left(l_{1}\right)}$ for some $\lambda \in \mathbb{R}$, and if $u \notin \operatorname{Ker}\left(l_{1}\right)$, then $l_{3}=\lambda l_{2}+\frac{l_{3}(u)-\lambda l_{2}(u)}{l_{1}(u)} l_{1}$ (because this is true on $\operatorname{Ker}\left(l_{1}\right)$ and on $u$ ). So, returning to our problem, $H \in \operatorname{Span}\left(P^{X}, P^{Y}\right)$ is equivalent to: $\operatorname{Ker}\left(P^{X^{*}}\right) \cap \operatorname{Ker}\left(P^{Y^{*}}\right) \subset$ $\operatorname{Ker}\left(H^{*}\right)$, where for any $L \in \mathbb{R}^{I}, L^{*}$ denotes the linear form defined by $L^{*}(y)=L \cdot y$. Let $x \in \operatorname{Ker}\left(\left(P^{X}\right)^{*}\right) \cap \operatorname{Ker}\left(\left(P^{Y}\right)^{*}\right)$. Clearly, there exists $\varepsilon>0$ such that $u^{I}+\varepsilon x$ and $u^{I}-\varepsilon x$ have non-negative coordinates, and so they are in $L_{U}$, and $H^{*}\left(u^{I}+\varepsilon x\right)=H^{*}\left(u^{I}-\varepsilon x\right)=e_{\max }$ otherwise one of them would be greater than $e_{\max }$, hence $x \in \operatorname{Ker}\left(H^{*}\right)$.

For instance, taking again $p^{X}=(2 / 3,1 / 6,1 / 6)$ and $p^{Y}=(1 / 6,2 / 3,1 / 6)$, we get $P^{X}=(3 / 2,6), P^{Y}=(6,3 / 2)$ and $s=t=2 / 15$.

Without loss of generality (switching the roles of $X$ and $Y$ ), one can thus assume that either Case a) or Case b) occurs.

In Case b), the following technical lemma, whose proof (given in the Appendix) is not crucial to understand the rest of this manuscript, is needed to state our main theorem. Let us define first, in Case b1),

$$
s_{X}:=\left\{\begin{array}{ll}
\max _{i \in I^{c}: p_{i}^{X} \geq e_{\max }} \frac{p_{i}^{Y}\left(p_{i}^{X}-e_{\max }\right)}{e_{\max }\left(p_{i}^{X}-p_{i}^{Y}\right)} & \text { if }\left\{i \in I^{c}, p_{i}^{X} \geq e_{\max }\right\} \neq \emptyset, \\
0, & \text { if }\left\{i \in I^{c}, p_{i}^{X} \geq e_{\max }\right\}=\emptyset,
\end{array} t_{X}:=1-s_{X},\right.
$$

and, similarly,

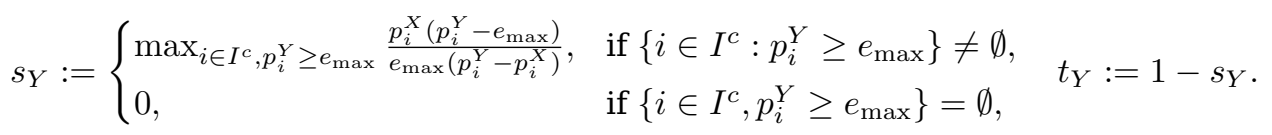

It is clear, from the definition of $I$, that if $i \in I$ is such that $p_{i}^{X} \geq e_{\max }$, then $p_{i}^{Y}<e_{\max }$, therefore $s_{X}$ and $s_{Y}$ are well defined and one can check that $s_{X}, t_{X}, s_{Y}, t_{Y} \in[0,1]$.

In order to state our next lemma, below let $E=\left\{x \in \mathbb{R}^{m}: x_{1}+\cdots+x_{m}=0\right\}$ and let $E^{\prime}=\left\{x \in E: \forall i \in I^{c}, x_{i} \geq 0\right\}$.

Lemma 1.5. Let $\nu \in\left(\mathbb{R}^{m}\right)^{2}$ be such that for all $i \in I^{c}, \nu_{i}^{X}=\nu_{i}^{Y}=0$, then the following maximum is well defined:

$$
\mathfrak{m}(\nu):=\max _{x \in E^{\prime 2}} \sum_{i=1}^{m}\left[\left(p_{i}^{X} x_{i}^{X}+\nu_{i}^{X}\right) \wedge\left(p_{i}^{Y} x_{i}^{Y}+\nu_{i}^{Y}\right)\right],
$$

and

$$
\mathfrak{m}(\nu)=\max _{\substack{x \in E^{\prime 2} \\\|x\|_{\infty} \leq 2 C m\|\nu\|_{\infty}}} \sum_{i=1}^{m}\left[\left(p_{i}^{X} x_{i}^{X}+\nu_{i}^{X}\right) \wedge\left(p_{i}^{Y} x_{i}^{Y}+\nu_{i}^{Y}\right)\right],
$$

for some constant $C>0$, depending only on $p^{X}$ and $p^{Y}$, as given in Lemma 2.3. In Case b1), writing $S^{\bullet}:=\sum_{i \in I} \nu_{i}^{\bullet}$, then

$$
\mathfrak{m}(\nu)=\left\{\begin{array}{ll}
s_{X} S^{Y}+t_{X} S^{X}, & \text { if } S^{X} \leq S^{Y} \\
s_{Y} S^{X}+t_{Y} S^{Y}, & \text { if } S^{X} \geq S^{Y}
\end{array} .\right.
$$

In Case b2), and recalling the notations of Lemma 1.4, then

$$
\mathfrak{m}(\nu)=\sum_{i \in I}\left(\frac{s}{p_{i}^{X}} \nu_{i}^{X}+\frac{t}{p_{i}^{Y}} \nu_{i}^{Y}\right) .
$$


On the limiting law of the length of the longest common and increasing subsequences

\subsection{Representation of $e_{\max }$}

We now aim to give a more explicit expression for $e_{\max }$ defined by (1.13). To do so, let us start with the following lemma which asserts that, in the non-probabilistic setup, "two letters are enough to reach the maximum".

Lemma 1.6. There exist $i, j \in\{1, \ldots, m\}$ and $\lambda \in K_{\Lambda^{2}}$ such that for all $k \notin\{i, j\}, \lambda_{k}^{X}=$ $\lambda_{k}^{Y}=0$.

Proof. Let $u \in L_{U}$ having (at least) three non-zero coordinates. Then, recalling the correspondence between $L_{U}$ and $K_{\Lambda^{2}}$, in order to prove the result it is enough to show that there exists a $v \in L_{U}$ having one less null coordinate. Without loss of generality, let $u_{1}, u_{2}, u_{3}>0$, and let

$$
V=\left\{x \in \mathbb{R}^{m}: \sum_{i=1}^{m} \frac{x_{i}}{p_{i}^{X}}=\sum_{i=1}^{m} \frac{x_{i}}{p_{i}^{Y}}=0, x_{4}=\cdots=x_{n}=0\right\} .
$$

Since the dimension of $V$ is at least one, let $x \in V \backslash\{0\}$. Then clearly, there exists $t \in \mathbb{R}$ such that $v:=u+t x$ has non-negative coordinates and one more null coordinate than $u$. Moreover, $v \in L_{U}$, which completes the proof.

If there exists $u \in L_{U}$ such all its coordinates except one, call it $i$, are zeros, then $e_{\max }=p_{i}^{X} \wedge p_{i}^{Y}$. Otherwise, let $i, j$ be defined as in the statement of the lemma. At first, assume that $p_{i}^{X}=p_{j}^{X}$ and that $p_{i}^{Y} \leq p_{j}^{Y}$, then $e_{\max } \leq\left(\lambda_{i}^{X} p_{i}^{X} \wedge \lambda_{i}^{Y} p_{j}^{Y}\right)+\left(\lambda_{j}^{X} p_{i}^{X} \wedge \lambda_{j}^{Y} p_{j}^{Y}\right) \leq$ $\left(\lambda_{i}^{X} p_{i}^{X}+\lambda_{j}^{X} p_{i}^{X}\right) \wedge\left(\lambda_{i}^{Y} p_{j}^{Y}+\lambda_{j}^{Y} p_{j}^{Y}\right)=p_{i}^{X} \wedge p_{i}^{Y}$, so $e_{\max }=p_{i}^{X} \wedge p_{i}^{Y}$ and we are actually in the first case, giving a contradiction. Similarly, if $p_{i}^{X} \leq p_{j}^{X}$ and $p_{i}^{Y} \leq p_{j}^{Y}$, using $\lambda_{i}^{X} p_{i}^{X} \wedge \lambda_{i}^{Y} p_{i}^{Y} \leq \lambda_{i}^{X} p_{j}^{X} \wedge \lambda_{i}^{Y} p_{j}^{Y}$ we get a contradiction as well. Therefore, in the second case, necessarily, possibly permuting $i$ and $j, p_{i}^{X}<p_{j}^{X}$ and $p_{i}^{Y}>p_{j}^{Y}$. Additionaly, it is necessary to have that $p_{i}^{X}<p_{i}^{Y}$, otherwise $e_{\max }=p_{i}^{Y}$ and we are in the first case. Similarly, $p_{j}^{Y}<p_{j}^{X}$. Then, in this case, the maximum is when the quantities in each minima are equal, and so one shows that

$$
e_{\max }=e(i, j):=\frac{p_{i}^{X} p_{i}^{Y}\left(p_{j}^{X}-p_{j}^{Y}\right)+p_{j}^{X} p_{j}^{Y}\left(p_{i}^{Y}-p_{i}^{X}\right)}{p_{i}^{Y} p_{j}^{X}-p_{i}^{X} p_{j}^{Y}} .
$$

Therefore,

$$
e_{\max }=\max \left(\max _{1 \leq i \leq m}\left(p_{i}^{X} \wedge p_{i}^{Y}\right), \max _{\substack{i, j: p_{i}^{X}<p_{j}^{X} \\ \wedge \\ p_{i}^{Y}>p_{j}^{Y}}} e(i, j)\right) .
$$

Note that

$$
\max _{1 \leq i \leq m}\left(p_{i}^{X} \wedge p_{i}^{Y}\right) \leq e_{\max } \leq\left(\max _{1 \leq i \leq m} p_{i}^{X}\right) \wedge\left(\max _{1 \leq i \leq m} p_{i}^{Y}\right),
$$

where the left inequality is clear, while the right one is easily seen from the expression of $f$. Note also that above, $e_{\max }$ is equal to the lower bound when the second max in (1.23) is over the empty set, and is equal to the upper bound when there exists $i$ such that $p_{\max }^{X}=p_{i}^{X} \leq p_{i}^{Y}$ or $p_{\max }^{Y}=p_{i}^{Y} \leq p_{i}^{X}$.

When $p^{X}=p^{Y}$ (same distribution for each word), we see that $e_{\max }=\max _{i \in\{1, \ldots, m\}} p_{i}^{X}$ is minimal when $p^{X}$ is uniform (for a given alphabet). This is to be contrasted with the case of the length of the longest common subsequences, $L C_{n}$ (defined just as $L C I_{n}$, but without the increasing condition). Indeed, little is known about $\gamma^{*}:=\lim _{n \rightarrow+\infty} \mathbb{E} L C_{n} / n$, for instance whether or not it is minimal (for a given alphabet) for the uniform distribution. Since $L C_{n}$ is defined with one less constraint than $L C I_{n}$, clearly $e_{\max } \leq \gamma^{*}$ which is of potential interest since the exact value of $\gamma^{*}$ is unknown, even in the binary uniform case. 
On the limiting law of the length of the longest common and increasing subsequences

(This last inequality provides a lower bound on $\gamma^{*}$, no matter the distributions on the letters. For uniform letters, $e_{\max }=1 / m$, although it is known that, then, asymptotically, $\gamma^{*} \sim 2 / \sqrt{m}$, see [7].)

\subsection{A criterion to distinguish the three cases}

For a given distribution, it is not completely apparent which situation is in play as far as the respective cases a), b1) and b2) are concerned. Our next result makes this more transparent. First, set

$$
e_{1}=\max _{1 \leq i \leq m}\left(p_{i}^{X} \wedge p_{i}^{Y}\right), \quad e_{2}=\max _{\substack{i, j: p_{i}^{X}<p_{j}^{X} \\ \hat{N} \in \\ p^{Y}>p^{Y}}} e(i, j),
$$

so that, by (1.23), $e_{\max }=\max \left(e_{1}, e_{2}\right)$.

Theorem 1.7. Let $e_{1}<e_{2}$, then Case b2) holds true. Let $e_{1} \geq e_{2}$, then:

(i) If for some $i \in\{1, \ldots, m\}$ such that $p_{i}^{X} \wedge p_{i}^{Y}=e_{1}$, one has $p_{i}^{X} \neq p_{i}^{Y}$, then Case a) holds true or so does its symmetric version: there exists $u \in L_{U}$ such that $\frac{u_{1}}{p_{1}^{Y}}+\cdots+\frac{u_{m}}{p_{m}^{Y}}=1$ and $\frac{u_{1}}{p_{1}^{X}}+\cdots+\frac{u_{m}}{p_{m}^{X}}<1$.

(ii) Otherwise, i.e., if for all $i \in\{1, \ldots, m\}$ such that $p_{i}^{X} \wedge p_{i}^{Y}=e_{1}$, one has $p_{i}^{X}=p_{i}^{Y}$, then if $e_{1}>e_{2}$ Case b1) holds true, while if $e_{1}=e_{2}$, then so does Case b2).

Proof. First, for any $0<\delta<1$, let $e_{\max , \delta}, e_{1, \delta}, e_{2, \delta}$ and $e_{\delta}(i, j)$ be defined just as $e_{\max }, e_{1}, e_{2}$ and $e(i, j)$ but replacing $p_{i}^{Y}$ with $\delta p_{i}^{Y}$, for all $i \in\{1, \ldots, m\}$. Next, from the very definition of Case a): There exists $u \in L_{U}$ such that $\frac{u_{1}}{p_{1}^{X}}+\cdots+\frac{u_{m}}{p_{m}^{X}}=1$ and $\frac{u_{1}}{p_{1}^{Y}}+\cdots+\frac{u_{m}}{p_{m}^{Y}}<1$. Letting $\delta_{0}:=\frac{u_{1}}{p_{1}^{Y}}+\cdots+\frac{u_{m}}{p_{m}^{Y}}$, we have $\frac{u_{1}}{\delta_{0} p_{1}^{Y}}+\cdots+\frac{u_{m}}{\delta_{0} p_{m}^{Y}}=1$ so $e_{\max , \delta_{0}} \geq e_{\max }$ and therefore (clearly, $e_{\max , \delta}$ is non-decreasing in $\delta$ ) $e_{\max , \delta_{0}}=e_{\max }$. So when Case a) occurs there exists $0<\delta_{0}<1$, such that for all $\delta \in\left(\delta_{0}, 1\right], e_{\max , \delta}=e_{\max }$, and one can easily check the converse. A similar result continues to hold for the symmetric version of Case a).

We can now prove the statement of the theorem by distinguishing the following four occurrences.

(1) Let $e_{1}<e_{2}$. Let $0<\delta_{0}<1$ be close enough to 1 such that for any $\delta \in\left(\delta_{0}, 1\right]$, the

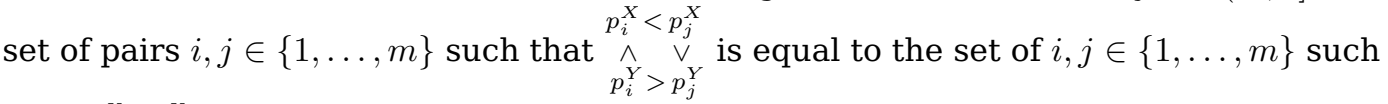
that $\underset{\delta p_{i}^{Y}>\delta p_{j}^{Y}}{\stackrel{p_{i}^{X}}{\wedge}}<p_{j}^{X}$. Since for every $i, j$ in this set, it is immediate to check that $e(i, j)>e_{\delta}(i, j)$, the maximums satisfy $e_{2}>e_{\delta, 2}$. Since $e_{1}<e_{2}$, by continuity, for $\delta$ close enough to 1 , $\max \left(e_{\delta, 1}, e_{\delta, 2}\right)=e_{\delta, 2}$ so $e_{\delta, \max }<e_{\max }$, hence we are in Case b). There are $i, j \in\{1, \ldots, m\}$ such that $e_{\max }=e_{2}=e(i, j)$, so $i, j$ are in $I$, but $p_{i}^{X}<p_{j}^{X}$ so we are in Case b2).

(2) Let $e_{1} \geq e_{2}$, and let there exist $i \in\{1, \ldots, m\}$ such that $p_{i}^{X} \wedge p_{i}^{Y}=e_{1}$ and $p_{i}^{X} \neq p_{i}^{Y}$, say, $p_{i}^{X}<p_{i}^{Y}$. Then, the very definition of Case a) is verified with the vector $u \in \mathbb{R}^{m}$ having coordinates equal to zero except for $u_{i}=p_{i}^{X}$. If instead, $p_{i}^{X}>p_{i}^{Y}$ then the symmetric case holds true.

(3) Let $e_{1}>e_{2}$ and let for all $i \in\{1, \ldots, m\}$ such that $p_{i}^{X} \wedge p_{i}^{Y}=e_{1}, p_{i}^{X}=p_{i}^{Y}$. By continuity, for $\delta$ close enough to $1, \max \left(e_{\delta, 1}, e_{\delta, 2}\right)=e_{\delta, 1}=\delta e_{\max }$ so we are in Case b). Additionally, one verifies that under our assumptions $I$ is restricted to the set of $i \in\{1, \ldots, m\}$ such that $p_{i}^{X}=p_{i}^{Y}=e_{\max }$. Therefore, we are, in fact, in Case b1).

(4) Let $e_{1}=e_{2}$ and let for all $i \in\{1, \ldots, m\}$ such that $p_{i}^{X} \wedge p_{i}^{Y}=e_{1}, p_{i}^{X}=p_{i}^{Y}$. From what is done above, we see that for $\delta$ close enough to $1, e_{\delta, \max }<e_{\max }$ hence we are in Case b). Once again, since there are $i, j \in\{1, \ldots, m\}$ such that $e_{\max }=e_{2}=e(i, j)$, we are in Case b2). 
On the limiting law of the length of the longest common and increasing subsequences

To present another explicit example, let us fully corner the case $m=2$, with $p_{1}^{X}, p_{2}^{X}, p_{1}^{Y}$, and $p_{2}^{Y}$. The following completely describes the various cases:

- If $p_{1}^{X}=p_{1}^{Y}$, then (since, necessarily, $\left.p_{2}^{X}=p_{2}^{Y}\right) e_{\max }=\max \left(p_{1}^{X}, p_{2}^{X}\right)=\max \left(p_{1}^{X}, 1-\right.$ $\left.p_{1}^{X}\right)$ and we are in Case b1).

- If $p_{1}^{X} \neq p_{1}^{Y}$ and $1 / 2 \in\left(\min \left(p_{1}^{X}, p_{1}^{Y}\right), \max \left(p_{1}^{X}, p_{1}^{Y}\right)\right)$, then

$$
e_{\text {max }}=\max \left(\min \left(p_{1}^{X}, p_{1}^{Y}\right), \min \left(p_{2}^{X}, p_{2}^{Y}\right)\right)=\max \left(\min \left(p_{1}^{X}, p_{1}^{Y}\right), \min \left(1-p_{1}^{X}, 1-p_{1}^{Y}\right)\right),
$$

and we are in Case a) or its symmetric.

- If $p_{1}^{X} \neq p_{1}^{Y}$ and $1 / 2 \notin\left(\min \left(p_{1}^{X}, p_{1}^{Y}\right), \max \left(p_{1}^{X}, p_{1}^{Y}\right)\right)$, then

$$
e_{\max }=\frac{p_{1}^{X} p_{1}^{Y}\left(p_{2}^{X}-p_{2}^{Y}\right)+p_{2}^{X} p_{2}^{Y}\left(p_{1}^{Y}-p_{1}^{X}\right)}{p_{1}^{Y} p_{2}^{X}-p_{1}^{X} p_{2}^{Y}}=p_{1}^{X} p_{1}^{Y}+p_{2}^{X} p_{2}^{Y}=p_{1}^{X} p_{1}^{Y}+\left(1-p_{1}^{X}\right)\left(1-p_{1}^{Y}\right),
$$

and we are in Case b2).

\section{The limiting law}

It is clear, from the previous section, that the proper way to center (and normalize) $L C I_{n}$ is via

$$
\begin{aligned}
Z_{n} & =\frac{L C I_{n}-n e_{\max }}{\sqrt{n}} \\
& =\max _{\lambda \in \Lambda^{2}} \sum_{i=1}^{m}\left[\left(\sqrt{n} p_{i}^{X} \lambda_{i}^{X}+\widetilde{V}_{i}^{n, X}\left(\lambda^{X}\right)\right) \wedge\left(\sqrt{n} p_{i}^{Y} \lambda_{i}^{Y}+\widetilde{V}_{i}^{n, Y}\left(\lambda^{Y}\right)\right)\right]-\sqrt{n} e_{\max } .
\end{aligned}
$$

Let also

$$
\begin{aligned}
Z_{n}^{c} & =\frac{L C I_{n}^{c}-n e_{\max }}{\sqrt{n}} \\
& =\max _{\lambda \in \Lambda^{2}} \sum_{i=1}^{m}\left[\left(\sqrt{n} p_{i}^{X} \lambda_{i}^{X}+V_{i}^{n, X}\left(\lambda^{X}\right)\right) \wedge\left(\sqrt{n} p_{i}^{Y} \lambda_{i}^{Y}+V_{i}^{n, Y}\left(\lambda^{Y}\right)\right)\right]-\sqrt{n} e_{\max },
\end{aligned}
$$

from (1.11) we have

$$
\left|Z_{n}-Z_{n}^{c}\right| \leq \frac{m}{\sqrt{n}}
$$

and therefore the convergence in distribution of $Z_{n}^{c}$ will imply the convergence, in distribution, of $Z_{n}$ towards the same limit.

\subsection{Statement of the theorem}

Below is the main result of the paper. In this statement, the covariance matrices of the Brownian motions stem from the covariance matrix of the rescaled variables $\left(\mathbb{1}_{X_{k}=i}\right)_{i \in I}$ (resp. $\mathbb{1}_{Y_{k}=i}, i \in I$ ) used to construct the polygonal approximations $B_{i}^{n, \bullet}$ (here, and throughout, $\bullet$ is short for either $X$ or $Y)$. Indeed, note that $\mathbb{E}\left(\frac{\left(\mathbb{1}_{X_{k}=i}-p_{i}^{X}\right)\left(\mathbb{1}_{X_{k}=j}-p_{j}^{X}\right)}{\sqrt{p_{i}^{X}\left(1-p_{i}^{X}\right)} \sqrt{p_{j}^{X}\left(1-p_{j}^{X}\right)}}\right)=$ $-\sqrt{\frac{p_{i}^{X} p_{j}^{X}}{\left(1-p_{i}^{X}\right)\left(1-p_{j}^{X}\right)}}($ with a similar result for $Y)$.

Theorem 2.1. Let $B^{X}$ and $B^{Y}$ be two independent $|I|$-dimensional Brownian motions defined on $[0,1]$ with respective covariance matrix $C^{X}$ defined by $C_{i, i}^{X}=1$ and $C_{i, j}^{X}=$ $-\sqrt{\frac{p_{i}^{X} p_{j}^{X}}{\left(1-p_{i}^{X}\right)\left(1-p_{j}^{X}\right)}}$, for $i \neq j$ in $I$, and $C^{Y}$ defined in a similar fashion, replacing $p_{i}^{X}$ by $p_{i}^{Y}$ 
On the limiting law of the length of the longest common and increasing subsequences

and $p_{j}^{X}$ by $p_{j}^{Y}$. For all $\lambda \in K_{\Lambda^{2}}$ and $i \in I$, set

$$
\begin{gathered}
V_{i}^{X}\left(\lambda^{X}\right)=\sqrt{p_{i}^{X}\left(1-p_{i}^{X}\right)}\left(B_{i}^{X}\left(\sum_{j=1}^{i} \lambda_{j}^{X}\right)-B_{i}^{X}\left(\sum_{j=1}^{i-1} \lambda_{j}^{X}\right)\right), \\
V_{i}^{Y}\left(\lambda^{Y}\right)=\sqrt{p_{i}^{Y}\left(1-p_{i}^{Y}\right)}\left(B_{i}^{Y}\left(\sum_{j=1}^{i} \lambda_{j}^{Y}\right)-B_{i}^{Y}\left(\sum_{j=1}^{i-1} \lambda_{j}^{Y}\right)\right) .
\end{gathered}
$$

If there exists $u \in L_{U}$ such that $\frac{u_{1}}{p_{1}^{X}}+\cdots+\frac{u_{m}}{p_{m}^{X}}=1$ and $\frac{u_{1}}{p_{1}^{Y}}+\cdots+\frac{u_{m}}{p_{m}^{Y}}<1$ (Case a)), then

$$
\frac{L C I_{n}-n e_{\max }}{\sqrt{n}} \underset{n \rightarrow \infty}{\Longrightarrow} Z^{a}:=\max _{\lambda^{X} \in J} \sum_{i \in I} V_{i}^{X}\left(\lambda^{X}\right)
$$

where $J$ is given by (1.16).

If for all $u \in L_{U}, \frac{u_{1}}{p_{1}^{X}}+\cdots+\frac{u_{m}}{p_{m}^{X}}=\frac{u_{1}}{p_{1}^{Y}}+\cdots+\frac{u_{m}}{p_{m}^{Y}}=1$ (Case b)), then

$$
\frac{L C I_{n}-n e_{\max }}{\sqrt{n}} \underset{n \rightarrow \infty}{\Longrightarrow} Z^{b}:=\max _{\lambda \in K_{\Lambda^{2}}} \mathfrak{m}\left(V^{X}\left(\lambda^{X}\right), V^{Y}\left(\lambda^{Y}\right)\right),
$$

where $\mathfrak{m}$ is given by (1.19).

At this point, one can remark that $e_{\max }$ is invariant with respect to the order in which the letters are chosen, and that both in Case a) and Case b1), the above limiting laws are invariant as well (to see this fact in Case a), recall Lemma 1.3). Therefore, in Case a) and Case b1), no matter the prescribed order (increasing, decreasing, etc..) the asymptotic behavior of the length of the corresponding optimal alignments is the same. We refer the reader to Section 3.2 for more general results of this flavor.

In Case b2) it is less clear that the limiting distribution is permutation-invariant as it might not just boil down to $\mathfrak{m}(\nu)$. Indeed, in Case b2) the limiting law can be written as the law of

$$
Z=\max _{\lambda \in K_{\Lambda^{2}}} \sum_{\substack{i \in\{1, \ldots, m\} \\ \bullet \in\{X, Y\}}} V(\lambda)_{i}^{\bullet},
$$

where $V(\lambda)$ is in $\left(\mathbb{R}^{m}\right)^{2}$, and defined via

$$
V^{\bullet}(\lambda)_{i}=B_{i}^{\bullet}\left(\sum_{j=1}^{i} \lambda_{j}^{\bullet}\right)-B_{i}^{\bullet}\left(\sum_{j=1}^{i-1} \lambda_{j}^{\bullet}\right),
$$

where the $B_{i}^{\bullet}$ are Brownian motions which are, up to a multiplicative factor, as in our main theorem. Further introducing, for any permutation $\sigma$ of $\{1, \ldots, n\}, V_{\sigma}(\lambda)$ defined via

$$
V_{\sigma}^{\bullet}(\lambda)_{i}=B_{i}^{\bullet}\left(\sum_{j=1}^{\sigma^{-1}(i)} \lambda_{\sigma(j)}^{\bullet}\right)-B_{i}^{\bullet}\left(\sum_{j=1}^{\sigma^{-1}(i)-1} \lambda_{\sigma(j)}^{\bullet}\right),
$$

we have $V(\lambda)=V_{\text {Id }}(\lambda)$, where Id is the identity permutation. When the letters are not required to be non-decreasing, but instead follow an order given by $\sigma$, the limiting law is simply the law of $Z_{\sigma}:=\max _{\lambda \in K_{\Lambda^{2}}} \sum_{\substack{i \in\{1, \ldots, m\} \\ \bullet \in\{X, Y\}}} V_{\sigma}(\lambda)_{i}^{\bullet}$. It is still not that clear whether or not this last quantity depends on $\sigma$. For example, if $m=3$ and $K_{\Lambda^{2}}=\Lambda^{2}$ and $B_{1}^{X}$ is a standard Brownian motion, while all others are null, define $\sigma$ by $\sigma(1)=2, \sigma(2)=$ $1, \sigma(3)=3$, then with probability one $Z_{\sigma}>Z_{\text {Id }}$. However, in Case b2) it is actually not possible to have $K_{\Lambda^{2}}=\Lambda^{2}$ (and also to have only one non null Brownian motion) but this shows that a general argument for the validity of the permutation-invariance is not that transparent. 
On the limiting law of the length of the longest common and increasing subsequences

\subsection{Proof of Theorem 2.1}

The proof of this theorem is based on a non-probabilistic lemma. First, let $E_{n}^{\eta}$ be the set of all continuous functions $b$ from $[0,1]$ into $\mathbb{R}$ such that: for all $x, y$ in $[0,1]$, $|b(y)-b(x)| \leq\left(n^{\eta} \sqrt{|y-x|}+n^{\eta-1 / 2}\right) / 2$. Then, for all $b \in\left(E_{n}^{\eta}\right)^{m}, i \in\{1, \ldots, m\}$ and $\lambda \in \Lambda$, set $v_{i}^{b}(\lambda)=b_{i}\left(\lambda_{1}+\cdots+\lambda_{i}\right)-b_{i}\left(\lambda_{1}+\cdots+\lambda_{i-1}\right)$, and for all $b^{X}, b^{Y} \in\left(E_{n}^{\eta}\right)^{m}$ and $\lambda \in \Lambda^{2}$ let

$$
z_{n}(\lambda)=\sum_{i=1}^{m}\left[\left(\sqrt{n} p_{i}^{X} \lambda_{i}^{X}+v_{i}^{b^{X}}\left(\lambda^{X}\right)\right) \wedge\left(\sqrt{n} p_{i}^{Y} \lambda^{Y}+v_{i}^{b^{Y}}\left(\lambda^{Y}\right)\right)\right]-\sqrt{n} e_{\max }
$$

One can think of $b_{i}^{X}$ (resp. $b_{i}^{Y}$ ) as $\sqrt{p_{i}^{X}\left(1-p_{i}^{X}\right)} B_{i}^{n, X}(\omega)\left(\operatorname{resp} . \sqrt{p_{i}^{Y}\left(1-p_{i}^{Y}\right)} B^{n, Y}(\omega)\right.$ ) for a fixed $\omega \in A_{n}^{\eta}$, where the symbol $b^{X}$ (resp. $b^{Y}$ ) is used for ease of notation and in order to emphasize the non-probabilistic nature of the proof. For further ease of notation, we omit the dependency in $b^{X}$ and $b^{Y}$ in the notation $z_{n}$. This omission is also present in $v$ and $v^{X}$ is just short for $v^{b^{X}}$ (similarly with $Y$ ), and further write $v(\lambda):=\left(v^{X}\left(\lambda^{X}\right), v^{Y}\left(\lambda^{Y}\right)\right)$. In Case a), for all $\lambda^{X} \in \Lambda$, let

$$
z^{a}\left(\lambda^{X}\right):=\sum_{i \in I} v_{i}^{X}\left(\lambda^{X}\right)
$$

In Case b), for all $\lambda \in \Lambda^{2}$, let

$$
z^{b}(\lambda)=\mathfrak{m}\left(v^{X}\left(\lambda^{X}\right), v^{Y}\left(\lambda^{Y}\right)\right)
$$

Next, let us finally present two simple inequalities stemming from the very definition of $E_{n}^{\eta}$, often used in the sequel, which are valid for all $b \in E_{n}^{\eta}, \lambda, \lambda^{\prime} \in \Lambda, i \in\{1, \ldots, m\}$, $\bullet \in\{X, Y\}$, namely,

$$
\begin{aligned}
\left|v_{i}^{\bullet}\left(\lambda^{\bullet}\right)\right| & \leq \frac{n^{\eta} \sqrt{\lambda_{i}^{\bullet}}+n^{\eta-1 / 2}}{2} \text { and in particular }\left|v_{i}^{\bullet}\left(\lambda^{\bullet}\right)\right| \leq n^{\eta}, \\
\left|v_{i}^{\bullet}\left(\lambda^{\bullet}\right)-v_{i}^{\bullet}\left(\lambda^{\prime \bullet}\right)\right| & \leq n^{\eta} \sqrt{\max _{i \in\{1, \ldots, m\}}\left|\lambda_{1}+\cdots+\lambda_{i}-\lambda_{1}^{\prime}-\cdots-\lambda_{i}^{\prime}\right|}+n^{\eta-1 / 2} \\
& \leq n^{\eta} \sqrt{m \mid\left\|\lambda-\lambda^{\prime}\right\|_{\infty}}+n^{\eta-1 / 2} .
\end{aligned}
$$

Lemma 2.2. There exists a sequence $\left(\varepsilon_{n}\right)_{n \geq 1}$ of positive reals converging to zero and such that for all $n \geq 1$ and $b^{X}, b^{Y} \in\left(E_{n}^{\eta}\right)^{m}$, either $\left|\max _{\lambda \in \Lambda^{2}} z_{n}(\lambda)-\max _{\lambda \in J} z^{a}(\lambda)\right| \leq \varepsilon_{n}$, or $\left|\max _{\lambda \in \Lambda^{2}} z_{n}(\lambda)-\max _{\lambda \in K_{\Lambda^{2}}} z^{b}(\lambda)\right| \leq \varepsilon_{n}$, in Case a) or b), respectively.

The proof of this crucial lemma is delayed to the next subsections, and instead we turn our attention to the proof of the main theorem.

Proof of Theorem 2.1. Let us assume that Case b) is occurring. Let

$$
Z_{n}^{b}=\max _{\lambda \in K_{\Lambda^{2}}} \mathfrak{m}\left(V^{n, X}\left(\lambda^{X}\right), V^{n, Y}\left(\lambda^{Y}\right)\right)
$$

For all $\omega \in A_{n}^{\eta}, B^{n, X}(\omega)$ and $B^{n, Y}(\omega)$ are in $E_{n}^{\eta}$ so by Lemma $2.2,\left|Z_{n}^{c}(\omega)-Z_{n}^{b}(\omega)\right| \leq \varepsilon_{n}$. So $\left|Z_{n}^{c}-Z_{n}^{b}\right| \mathbb{1}_{A_{n}^{\eta}} \leq \varepsilon_{n}$, but $Z_{n}^{c}-Z_{n}^{b}=\left(Z_{n}^{c}-Z_{n}^{b}\right) \mathbb{1}_{A_{n}^{\eta}}+\left(Z_{n}^{c}-Z_{n}^{b}\right) \mathbb{1}_{\left(A_{n}^{\eta}\right)^{c}}$, where this second term tends to zero in probability, therefore so does $Z_{n}^{c}-Z_{n}^{b}$. Next, by Donsker's theorem and the continuity of $\mathfrak{m}$ (recalling Lemma 1.5), $Z_{n}^{b}$ tends to $Z^{b}$ in distribution, so does $Z_{n}^{c}$ and finally so is the case for $Z_{n}$, recalling (2.1). The proof in the Case a) is analogous and therefore omitted. 
On the limiting law of the length of the longest common and increasing subsequences

Let us now turn to the proof of Lemma 2.2. The method of proof goes as follows: Maximizing $z_{n}(\lambda)$ is equivalent to maximizing

$$
z_{n}(\lambda) / \sqrt{n}=\sum_{i=1}^{m}\left[\left(p_{i}^{X} \lambda_{i}^{X}+v_{i}^{b^{X}}\left(\lambda^{X}\right) / \sqrt{n}\right) \wedge\left(p_{i}^{Y} \lambda^{Y}+v_{i}^{b^{Y}}\left(\lambda^{Y}\right) / \sqrt{n}\right)\right]-e_{\max }
$$

which converges, as $n$ goes to infinity, to $f(\lambda)-e_{\max }$. So one can expect that $\lambda$ must "almost" be maximizing $f$, i.e., be in or "close to" the set $K_{\Lambda^{2}}$. In Case a), we bound the maximum by taking the maximum over two sets which are closer and closer to the set $J$. In Case b), first write $\lambda=\lambda^{K_{\Lambda^{2}}}+\lambda^{r}$ (actually dealing with a $\lambda-a$ in order to have a vector space, but the idea is the same), then ignore the small perturbation term $\lambda^{r}$ in $v$, and the idea is (roughly) to fix $\lambda^{K_{\Lambda^{2}}}$ and to find the maximum over $\lambda^{r}$. In both cases, the end of the proof consists in showing how the maximum of the relevant function $\left(z^{a}\right.$ or $\left.z^{b}\right)$ over a set of parameters that "tends to" a limiting set goes to the maximum over this limiting set.

\subsection{Proof of Lemma 2.2, Case a)}

\subsubsection{Restriction to $I$}

First, fix $b=\left(b^{X}, b^{Y}\right) \in\left(\left(E_{n}^{\eta}\right)^{m}\right)^{2}$. Next, for ease of notation, omit in the sub-index $b$ in $z$ and $v$. Roughly speaking, we begin by proving that any $\lambda$ maximizing $z_{n}$ must have "small" coordinates outside of $I$, and therefore we can "replace" the variations $v_{i}$, for $i \notin I$, by zero.

Let

$$
p_{\mathrm{sec}}^{X}=\left\{\begin{array}{ll}
\max _{i \notin I} p_{i}^{X} & I \neq\{1, \ldots, m\} \\
0 & I=\{1, \ldots, m\}
\end{array} .\right.
$$

Let us assume first that $I \neq\{1, \ldots, m\}$. Then by Lemma 1.3, $p_{\mathrm{sec}}^{X}<p_{\max }^{X}$. Our first observation is that if $\lambda$ maximizes $z_{n}$, i.e., if $z_{n}(\lambda)=\max _{\lambda \in \Lambda^{2}} z_{n}(\lambda)$, then

$$
s:=\sum_{i \notin I} \lambda_{i}^{X} \leq \frac{2 m n^{\eta-1 / 2}}{p_{\max }^{X}-p_{\mathrm{sec}}^{X}}
$$

In words, the above indicates that the contribution of the letters not in $I$ is, as expected, very limited. To prove this inequality, note that on the one hand (recalling Lemma 1.3 and (2.6)),

$$
z_{n}(\lambda) \leq \sum_{i=1}^{m}\left(\sqrt{n} p_{i}^{X} \lambda_{i}^{X}+v_{i}^{b}(\lambda)\right)-\sqrt{n} p_{\max }^{X} \leq \sqrt{n}\left(p_{\max }^{X}(1-s)+p_{\mathrm{sec}}^{X} s\right)+m n^{\eta}-\sqrt{n} p_{\max }^{X},
$$

while on the other hand, for $\tilde{\lambda} \in K_{\Lambda^{2}}$, using (2.6) and the elementary inequality (1.10),

$$
z_{n}(\lambda) \geq z_{n}(\tilde{\lambda}) \geq \sqrt{n} f(\tilde{\lambda})-m n^{\eta}-\sqrt{n} p_{\max }^{X}=-m n^{\eta}
$$

The inequality (2.10) follows, and it therefore allows, for $i \notin I$, to replace the terms $v_{i}^{X}\left(\lambda^{X}\right)$ by zero. More precisely, let for all $\lambda \in \Lambda^{2}$,

$$
\begin{aligned}
z_{n}^{I}(\lambda)=\sum_{i \in I}\left[\left(\sqrt{n} p_{i}^{X} \lambda_{i}^{X}+v_{i}^{X}\left(\lambda^{X}\right)\right) \wedge\left(\sqrt{n} p_{i}^{Y} \lambda_{i}^{Y}+v_{i}^{Y}\left(\lambda^{Y}\right)\right)\right] \\
+\sum_{i \notin I}\left[\left(\sqrt{n} p_{i}^{X} \lambda_{i}^{X}\right) \wedge\left(\sqrt{n} p_{i}^{Y} \lambda_{i}^{Y}+v_{i}^{Y}\left(\lambda^{Y}\right)\right)\right]-\sqrt{n} e_{\max }
\end{aligned}
$$


On the limiting law of the length of the longest common and increasing subsequences

then as shown next,

$$
\left|\max _{\lambda \in \Lambda^{2}} z_{n}(\lambda)-\max _{\lambda \in \Lambda^{2}} z_{n}^{I}(\lambda)\right| \leq \frac{\left|I^{c}\right|}{2}\left(n^{\eta} \sqrt{\frac{2 m n^{\eta-1 / 2}}{p_{\max }^{X}-p_{\text {sec }}^{X}}}+n^{\eta-1 / 2}\right),
$$

and this inequality remains true when $I=\{1, \ldots, m\}$ (since then $\max _{\lambda \in \Lambda^{2}} z_{n}(\lambda)=$ $\max _{\lambda \in \Lambda^{2}} z_{n}^{I}(\lambda)$ and $\left|I^{c}\right|=0$ ).

Indeed, let $\lambda \in \Lambda^{2}$ be such that $z_{n}(\lambda)=\max _{\lambda \in \Lambda^{2}} z_{n}(\lambda)$. Using (1.10) along with (2.6) $\left(\lambda_{i}^{X} \leq 2 m n^{\eta-1 / 2} /\left(p_{\max }^{X}-p_{\mathrm{sec}}^{X}\right)\right.$, for all $\left.i \notin I\right)$, it follows that

$$
\max _{\lambda \in \Lambda^{2}} z_{n}^{I}(\lambda) \geq z_{n}^{I}(\lambda) \geq \max _{\lambda \in \Lambda^{2}} z_{n}(\lambda)-\frac{\left|I^{c}\right|}{2}\left(n^{\eta} \sqrt{\frac{2 m n^{\eta-1 / 2}}{p_{\max }^{X}-p_{\text {sec }}^{X}}}+n^{\eta-1 / 2}\right) .
$$

Moreover, let $\tilde{\lambda} \in \Lambda^{2}$ be such that $\max _{\lambda \in \Lambda^{2}} z_{n}^{I}(\lambda)=z_{n}^{I}(\tilde{\lambda})$. Then, just as in proving (2.10), it follows that $\sum_{i \notin I} \tilde{\lambda}_{i}^{X} \leq 2|I| n^{\eta-1 / 2} /\left(p_{\max }^{X}-p_{\text {sec }}^{X}\right)$. Hence

$$
\max _{\lambda \in \Lambda^{2}} z_{n}(\lambda) \geq z_{n}(\tilde{\lambda}) \geq \max _{\lambda \in \Lambda^{2}} z_{n}^{I}(\lambda)-\frac{\left|I^{c}\right|}{2}\left(n^{\eta} \sqrt{\frac{2 m n^{\eta-1 / 2}}{p_{\max }^{X}-p_{\text {sec }}^{X}}}+n^{\eta-1 / 2}\right),
$$

which completes the proof.

\subsubsection{Bounds on the maximum with different sets of constraints}

Let us next define two sets "close" to $J$. To do so, let $S_{n}=2|I|^{2} n^{\eta-1 / 2}$, let $C_{I}=\sum_{i \in I} \frac{1}{p_{i}^{Y}}$, let $T_{n}=C_{I} 2 n^{\eta-1 / 2}$, and finally let

$$
J_{n}^{+}=\left\{\lambda^{X} \in \Lambda: \sum_{i \in I} \frac{\lambda_{i}^{X}}{p_{i}^{Y}} \leq \frac{1+S_{n}}{p_{\max }^{X}}\right\},
$$

and

$$
J_{n}^{-}=\left\{\lambda^{X} \in \Lambda: \sum_{i \in I} \frac{\lambda_{i}^{X}}{p_{i}^{Y}} \leq \frac{1-T_{n}}{p_{\max }^{X}}\right\} .
$$

Note that by Lemma 1.3, setting $\delta_{i_{1}}=\left(\mathbb{1}_{i=i_{1}}\right)_{i \in\{1, \ldots, m\}}, \delta_{i_{1}} \in J_{n}^{-}$eventually. We show, in this part of the proof, that

$$
\max _{\lambda \in J_{n}^{-}} z^{a}(\lambda) \leq \max _{\lambda \in \Lambda^{2}} z_{n}^{I}(\lambda) \leq \max _{\lambda \in J_{n}^{+}} z^{a}(\lambda) .
$$

Let us prove the upper bound first. Let $\lambda \in \Lambda^{2}$ be such that $z_{n}^{I}(\lambda)=\max _{\lambda \in \Lambda^{2}} z_{n}^{I}(\lambda)$, and let $S$ be the unique real such that

$$
\sum_{i \in I} \frac{\lambda_{i}^{X}}{p_{i}^{Y}}=\frac{1+S}{p_{\max }^{X}} .
$$

Then, there exists $i_{0} \in I$ such that,

$$
\lambda_{i_{0}}^{Y} p_{i_{0}}^{Y} \leq \lambda_{i_{0}}^{X} p_{\max }^{X}-\frac{S}{|I|},
$$

since otherwise, $\sum_{i \in I} \lambda_{i}^{Y}>1$, which is a contradiction. Then, using the following inequalities,

$$
\begin{gathered}
\forall i \in I \backslash\left\{i_{0}\right\} \quad\left(\sqrt{n} p_{i}^{X} \lambda_{i}^{X}+v_{i}^{X}\left(\lambda^{X}\right)\right) \wedge\left(\sqrt{n} p_{i}^{Y} \lambda_{i}^{Y}+v_{i}^{Y}\left(\lambda^{Y}\right)\right) \leq\left(\sqrt{n} p_{i}^{X} \lambda_{i}^{X}+v_{i}^{X}\left(\lambda^{X}\right)\right), \\
\left(\sqrt{n} p_{i_{0}}^{X} \lambda_{i_{0}}^{X}+v_{i_{0}}^{X}\left(\lambda^{X}\right)\right) \wedge\left(\sqrt{n} p_{i_{0}}^{Y} \lambda_{i_{0}}^{Y}+v_{i_{0}}^{Y}\left(\lambda^{Y}\right)\right) \leq\left(\sqrt{n}\left(\lambda_{i_{0}}^{X} p_{\max }^{X}-\frac{S}{|I|}\right)+v_{i_{0}}^{Y}\left(\lambda^{Y}\right)\right), \\
\forall i \notin I \quad\left(\sqrt{n} p_{i}^{X} \lambda_{i}^{X}\right) \wedge\left(\sqrt{n} p_{i}^{Y} \lambda_{i}^{Y}+v_{i}^{Y}\left(\lambda^{Y}\right)\right) \leq \sqrt{n} p_{i}^{X} \lambda_{i}^{X},
\end{gathered}
$$


On the limiting law of the length of the longest common and increasing subsequences

leads to

$$
\begin{aligned}
z_{n}^{I}(\lambda) & \leq \sqrt{n} \sum_{i=1}^{m} p_{i}^{X} \lambda_{i}^{X}+\sum_{i \in I \backslash\left\{i_{0}\right\}}\left(v_{i}^{X}\left(\lambda^{X}\right)+v_{i_{0}}^{Y}\left(\lambda^{Y}\right)\right)-\sqrt{n} \frac{S}{|I|}-\sqrt{n} e_{\max } \\
& \leq \sum_{i \in I \backslash\left\{i_{0}\right\}}\left(v_{i}^{X}\left(\lambda^{X}\right)+v_{i_{0}}^{Y}\left(\lambda^{Y}\right)\right)-\sqrt{n} \frac{S}{|I|} \\
& \leq|I| n^{\eta}-\sqrt{n} \frac{S}{|I|} .
\end{aligned}
$$

Just as in obtaining the inequality (2.11), we have $-|I| n^{\eta} \leq z_{n}^{I}(\lambda)$, hence $S \leq 2|I|^{2} n^{\eta-1 / 2}$, i.e., $\lambda^{X} \in J_{n}^{+}$, leading to conclude with the upper estimate:

$$
\max _{\lambda \in \Lambda^{2}} z_{n}^{I}(\lambda)=z_{n}^{I}(\lambda) \leq \sqrt{n} f\left(\lambda^{X}\right)+z^{a}\left(\lambda^{X}\right)-\sqrt{n} e_{\max } \leq z^{a}\left(\lambda^{X}\right) \leq \max _{\lambda \in J_{n}^{+}} z^{a}(\lambda) .
$$

Let us now turn our attention to the lower bound. Let $\lambda^{X} \in J_{n}^{-}$be such that $z^{a}\left(\lambda^{X}\right)=\max _{\lambda \in J_{n}^{-}} z^{a}(\lambda)$. Since

$$
\sum_{i \in I}\left(p_{\max }^{X} \lambda_{i}^{X}+2 n^{\eta-1 / 2}\right) / p_{i}^{Y} \leq 1
$$

there exists $\lambda^{Y} \in \Lambda$ such that for $i \in I, \lambda_{i}^{Y} \geq\left(p_{\max }^{X} \lambda_{i}^{X}+2 n^{\eta-1 / 2}\right) / p_{i}^{Y}$ and for $i \notin I$, $\lambda_{i}^{Y}=0$. For all $i \in I$,

$$
\begin{aligned}
\sqrt{n} p_{i}^{Y} \lambda_{i}^{Y}+v_{i}^{Y}\left(\lambda^{Y}\right) & \geq \sqrt{n} p_{\max }^{X} \lambda_{i}^{X}+2 n^{\eta}+v_{i}^{Y}\left(\lambda^{Y}\right) \\
& \geq \sqrt{n} p_{\max }^{X} \lambda_{i}^{X}+v_{i}^{X}\left(\lambda^{X}\right)=\sqrt{n} p_{i}^{X} \lambda_{i}^{X}+v_{i}^{X}\left(\lambda^{X}\right) .
\end{aligned}
$$

Therefore,

$$
\begin{aligned}
z_{n}^{I}(\lambda) & =\sum_{i \in I}\left(\sqrt{n} p_{i}^{X} \lambda_{i}^{X}+v_{i}^{X}\left(\lambda^{X}\right)\right)+\sum_{i \notin I}\left[\left(\sqrt{n} p_{i}^{X} \lambda_{i}^{X}\right) \wedge 0\right]-\sqrt{n} p_{\max }^{X} \\
& =\sum_{i \in I} v_{i}^{X}\left(\lambda^{X}\right)=z^{a}\left(\lambda^{X}\right)=\max _{\lambda \in J_{n}^{-}} z^{a}(\lambda)
\end{aligned}
$$

and $\max _{\lambda \in J_{n}^{-}} z^{a}(\lambda) \leq \max _{\lambda \in \Lambda^{2}} z_{n}^{I}(\lambda)$.

\subsubsection{End of the proof}

Both quantities $\left|\max _{\lambda \in J_{n}^{-}} z^{a}(\lambda)-\max _{\lambda \in J} z^{a}(\lambda)\right|$ and $\left|\max _{\lambda \in J_{n}^{+}} z^{a}(\lambda)-\max _{\lambda \in J} z^{a}(\lambda)\right|$ still need to be investigated. Let $C_{1}=\left(1-\frac{p_{\max }^{X}}{p_{i_{1}}^{X}}\right)>0$. For $\lambda^{X} \in \Lambda$ and $t \in(0,1)$, let $\lambda^{X, t}=t \delta_{i_{1}}+(1-t) \lambda^{X}$. It is straightforward to prove that for all $n$ greater than some constant, depending only on $\eta, p^{X}$ and $p^{Y}$, and for all $\lambda^{X} \in J, \lambda^{X, \frac{T_{n}}{C_{1}}}$ is well defined, and is in $J_{n}^{-}$, while for all $\lambda^{X} \in J_{n}^{+}, \lambda^{X, \frac{2 S_{n}}{C_{1}}} \in J$.

This is useful since for all $i \in\{1, \ldots, m\}$,

$$
\left|\lambda_{1}^{X}+\cdots+\lambda_{i}^{X}-\lambda_{1}^{X, t}-\cdots-\lambda_{i}^{X, t}\right| \leq 2 t
$$

and therefore, using (1.10) along with (2.7),

$$
\begin{aligned}
& \max _{\lambda \in J} z^{a}(\lambda)-\max _{\lambda \in J_{n}^{-}} z^{a}(\lambda) \leq|I|\left(n^{\eta} \sqrt{\frac{2 T_{n}}{C_{1}}}+n^{\eta-1 / 2}\right), \\
& \max _{\lambda \in J_{n}^{+}} z^{a}(\lambda)-\max _{\lambda \in J} z^{a}(\lambda) \leq|I|\left(n^{\eta} \sqrt{\frac{4 S_{n}}{C_{1}}}+n^{\eta-1 / 2}\right) .
\end{aligned}
$$


On the limiting law of the length of the longest common and increasing subsequences

Putting these two inequalities, together with (2.13), leads to

$$
\left|\max _{\lambda \in \Lambda^{2}} z_{n}^{I}(\lambda)-\max _{\lambda \in J} z^{a}(\lambda)\right| \leq C_{2} n^{\frac{6 \eta-1}{4}}+|I| n^{\eta-1 / 2},
$$

for some constant $C_{2}$ depending only on the $p^{\prime}$ s but need not be made explicit. The lemma is thus proved in this case.

\subsection{Proof of Lemma 2.2, Case b)}

\subsubsection{Preliminaries}

Fix $b=\left(b^{X}, b^{Y}\right) \in\left(\left(E_{n}^{\eta}\right)^{m}\right)^{2}$. Just as in Case a), we omit in the notation the sub-index $b$. Let $E=\left\{x \in \mathbb{R}^{m}: x_{1}+\cdots+x_{m}=0\right\}$, let $K$ be the subspace of $E^{2}$ defined by

$$
K=\left\{x \in E^{2}: \forall i \in I, p_{i}^{X} x_{i}^{X}=p_{i}^{Y} x_{i}^{Y}, \forall i \notin I, x_{i}^{X}=y_{i}^{Y}=0\right\},
$$

and let $P$ (recalling the definition of $a$ following (1.15): $a \in K_{\Lambda^{2}}$, for all $i \in I, p_{i}^{X} a_{i}^{X}=$ $p_{i}^{Y} a_{i}^{Y}>0$, for $i \notin I, a_{i}^{\bullet}=0$, and $f(a)=e_{\max }$ ) be given by:

$$
P=\left\{x \in E^{2}: \forall i \in\{1, \ldots, m\}, x_{i}^{X} \geq-a_{i}^{X}, x_{i}^{Y} \geq-a_{i}^{Y}\right\} .
$$

Note that $\Lambda^{2}=a+P$. By definition of the case b), for all $\lambda \in K_{\Lambda^{2}}$, for all $i \in I$ $\lambda_{i}^{X} p_{i}^{X}=\lambda_{i}^{Y} p_{i}^{Y}$, while for all $i \notin I, \lambda_{i}^{X}=\lambda_{i}^{Y}=0$. Reciprocally, let $\lambda \in \Lambda^{2}$ such that for all $i \in I \lambda_{i}^{X} p_{i}^{X}=\lambda_{i}^{Y} p_{i}^{Y}$ and for all $i \notin I, \lambda_{i}^{X}=\lambda_{i}^{Y}=0$, we show that $\lambda \in K_{\Lambda^{2}}$. Let $u \in \mathbb{R}^{I}$ be defined by $u_{i}=p_{i}^{X} \lambda_{i}^{X}-p_{i}^{X} a_{i}^{X}$ for all $i \in I$. We have that $u \cdot P^{X}=u \cdot P^{Y}=1-1=0$ so by Lemma $1.4, u \cdot(1)_{i \in I}=0$, hence the result. This characterization of $K_{\Lambda^{2}}$, combined with $\Lambda^{2}=a+P$, gives us

$$
K_{\Lambda^{2}}=a+K \cap P .
$$

Since $p_{i}^{X} a_{i}^{X}=p_{i}^{Y} a_{i}^{Y}$, for all $i \in\{1, \ldots, m\}$,

$$
z_{n}(a+x)=\sum_{i=1}^{m}\left[\left(\sqrt{n} p_{i}^{X} x_{i}^{X}+v_{i}^{X}\left(a^{X}+x^{X}\right)\right) \wedge\left(\sqrt{n} p_{i}^{Y} x_{i}^{Y}+v_{i}^{Y}\left(a^{Y}+x^{Y}\right)\right)\right] .
$$

Clearly,

$$
\max _{\lambda \in \Lambda^{2}} z_{n}(\lambda)=\max _{x \in P} z_{n}(a+x)
$$

Note also that for all $x \in\left(\mathbb{R}^{m}\right)^{2}, f(a+x)=f(a)+f(x)$ so by (2.15)

$$
\forall x \in P, f(x) \leq 0 \quad \text { and } \quad(f(x)=0) \Longleftrightarrow(x \in K \cap P) .
$$

Our next result is an elementary projection result.

Lemma 2.3. There exists $C>0$ depending only on $p^{X}$ and $p^{Y}$ such that for all $x \in P$, there exist $x^{K \cap P} \in K \cap P$ and $x^{r} \in E^{2}$ such that $x=x^{K \cap P}+x^{r}$ and $\left\|x^{r}\right\|_{\infty} \leq-C f(x)$.

Proof. Let $K^{\perp}$ be the orthogonal complement of $K$ in $E^{2}$ (for the usual Euclidean inner product defined on $E^{2}$ by, for $x, y \in E^{2}, x \cdot y:=x_{1}^{X} y_{1}^{X}+\cdots+x_{m}^{X} y_{m}^{X}+x_{1}^{Y} y_{1}^{Y}+\cdots+x_{m}^{Y} y_{m}^{Y}$ ). Let $x \in P$ (so $x \in E^{2}$ ) and let $\left(x^{K}, x^{K^{\perp}}\right)$ be its orthogonal decomposition, i.e., $x^{K} \in K$, $x^{K^{\perp}} \in K^{\perp}$ and $x=x^{K}+x^{K^{\perp}}$. Without loss of generality, assume $x^{K^{\perp}} \neq 0$. For ease of notation, set $g=-f$. Let

$$
a_{\min }=\min _{i \in I} a_{i}
$$

In order to bound the image of $x^{K^{\perp}}$, we first rescale it to make it an element of $P$ : it is easy to check that $y:=\left(\frac{a_{\min }}{\left\|x^{K^{\perp}}\right\|_{\infty}}\right) x^{K^{\perp}} \in P$. Now, consider the sphere,

$$
S_{a_{\min }}:=\left\{z \in K^{\perp}:\|z\|_{\infty}=a_{\min }\right\} .
$$


On the limiting law of the length of the longest common and increasing subsequences

Then, $S_{a_{\min }} \cap P$ is a non-empty compact set, so let

$$
M=\min _{z \in S_{a_{\min }} \cap P} g(z) .
$$

Recalling (2.16), $M>0$. Since $y \in S_{a_{\min }} \cap P, M \leq g(y)$ so that, using $g\left(x^{K^{\perp}}\right)=g(x)$,

$$
\left\|x^{K^{\perp}}\right\|_{\infty} \leq \frac{a_{\min }}{M} g(x) .
$$

This is almost the desired result, except that $x^{K}$ might not be in $P$. Let us assume, firstly, that $g(x) \leq M$ (and therefore that $\left\|x^{K^{\perp}}\right\|_{\infty} \leq a_{\min }$ ). Let $x^{K \cap P}=\left(1-\frac{\left\|x^{K^{\perp}}\right\|_{\infty}}{a_{\min }}\right) x^{K}$ and let $x^{r}=\frac{\left\|x^{K^{\perp}}\right\|_{\infty}}{a_{\min }} x^{K}+x^{K^{\perp}}$. We next prove that $x^{K \cap P} \in K \cap P$. Since $x \in P$, for $i \in I$,

$$
\begin{aligned}
&\left(1-\frac{\left\|x^{K^{\perp}}\right\|_{\infty}}{a_{\min }}\right) x_{i}^{K}+\left(1-\frac{\left\|x^{K^{\perp}}\right\|_{\infty}}{a_{\min }}\right) x_{i}^{K^{\perp}} \geq-\left(1-\frac{\left\|x^{K^{\perp}}\right\|_{\infty}}{a_{\min }}\right) a_{i} \\
& x_{i}^{K \cap P} \geq-a_{i}+\frac{\left\|x^{K^{\perp}}\right\|_{\infty}}{a_{\min }} a_{i}-\left(1-\frac{\left\|x^{K^{\perp}}\right\|_{\infty}}{a_{\min }}\right) x_{i}^{K^{\perp}} \\
& \geq-a_{i}+\left\|x^{K^{\perp}}\right\|_{\infty}-\left(1-\frac{\left\|x^{K^{\perp}}\right\|_{\infty}}{a_{\min }}\right)\left\|x^{K^{\perp}}\right\|_{\infty} \\
& \geq-a_{i},
\end{aligned}
$$

and for $i \notin I, x_{i}^{K \cap P}=0$, since $x^{K \cap P} \in K$. So $x^{K \cap P} \in K \cap P$.

Let us turn to $x^{r}$. Since $a+x \in \Lambda^{2},\|x\|_{\infty} \leq 1$. Moreover, $x^{K}$ is the orthogonal projection of $x$ so $\left\|x^{K}\right\|_{\infty} \leq \sqrt{2 m}\|x\|_{\infty} \leq \sqrt{2 m}$ and

$$
\begin{aligned}
\left\|x^{r}\right\|_{\infty} & \leq\left(\frac{\sqrt{2 m}}{a_{\min }}+1\right)\left\|x^{K^{\perp}}\right\|_{\infty} \\
& \leq\left(\frac{\sqrt{2 m}}{a_{\min }}+1\right) \frac{a_{\min }}{M} g(x) .
\end{aligned}
$$

Setting $C:=\left(\sqrt{2 m}+a_{\min }\right) / M$, we have just proved that if $g(x) \leq M$, then there exist suitable $x^{K \cap P}$ and $x^{r}$ satisfying the lemma. Finally, if $g(x)>M$, we let $x^{K \cap P}=0$ and $x^{r}=x$, so that $\left\|x^{r}\right\|_{\infty} \leq 1<g(x) / M<C g(x)$ which completes the proof.

\subsubsection{Separation of the parameters}

To begin with, we prove that $\max _{x \in P} z_{n}(a+x)$ can be written as a maximum over two kind of parameters, one belonging to $K$ in the variations $v_{i}$, the other one being a small remaining term.

Let $x \in P$ be such that $z_{n}(a+x)=\max _{\lambda \in \Lambda^{2}} z_{n}(\lambda)$. Then,

$$
-m n^{\eta} \leq z_{n}(a) \leq z_{n}(a+x) \leq \sqrt{n} f(x)+m n^{\eta},
$$

and so

$$
-f(x) \leq 2 m n^{\eta-1 / 2}
$$

Now, let

$$
D=\left\{\left(x^{K \cap P}, x^{r}\right) \in(K \cap P) \times E^{2}: x^{K \cap P}+x^{r} \in P\right\},
$$


On the limiting law of the length of the longest common and increasing subsequences

and, recalling the constant $C$ from Lemma 2.3, let

$$
D_{n}=\left\{\left(x^{K \cap P}, x^{r}\right) \in(K \cap P) \times E^{2}:\left\|x^{r}\right\|_{\infty} \leq 2 C m n^{\eta-1 / 2}, x^{K \cap P}+x^{r} \in P\right\} .
$$

Then, for all $\left(x^{K \cap P}, x^{r}\right) \in D$, set

$$
\begin{aligned}
& \bar{z}_{n}\left(x^{K \cap P}, x^{r}\right)=z_{n}\left(a+x^{K \cap P}+x^{r}\right)= \\
& \sum_{i=1}^{m}\left[\left(\sqrt{n} p_{i}^{X} x_{i}^{r, X}+v_{i}^{X}\left(a^{X}+x^{K \cap P, X}+x^{r, X}\right)\right) \wedge\left(\sqrt{n} p_{i}^{Y} x_{i}^{r, Y}+v_{i}^{Y}\left(a^{Y}+x^{K \cap P, Y}+x^{r, Y}\right)\right)\right] .
\end{aligned}
$$

Applying Lemma 2.3 to (2.17) gives $\max _{x \in D_{n}} \bar{z}_{n}(x)=\max _{x \in P} z_{n}(a+x)$.

Let us next define a slight modification of $\bar{z}_{n}$ by letting, for all $\left(x^{K \cap P}, x^{r}\right) \in D_{n}$,

$$
\bar{z}_{n}^{\prime}\left(x^{K \cap P}, x^{r}\right)=\sum_{i=1}^{m}\left[\left(\sqrt{n} p_{i}^{X} x_{i}^{r, X}+v_{i}^{X}\left(a^{X}+x^{K \cap P, X}\right)\right) \wedge\left(\sqrt{n} p_{i}^{Y} x_{i}^{r, Y}+v_{i}^{Y}\left(a^{Y}+x^{K \cap P, Y}\right)\right)\right] .
$$

The parameters are now "separated". For all $\left(x^{K \cap P}, x^{r}\right) \in D_{n}$, by (2.7),

$$
\left|\bar{z}_{n}^{\prime}\left(x^{K \cap P}, x^{r}\right)-\bar{z}_{n}\left(x^{K \cap P}, x^{r}\right)\right| \leq m\left(n^{\eta} \sqrt{2 C m^{2} n^{\eta-1 / 2}}+n^{\eta-1 / 2}\right),
$$

so that

$$
\begin{aligned}
\left|\max _{x \in P} z_{n}(a+x)-\max _{x \in D_{n}} \bar{z}_{n}^{\prime}(x)\right| & =\left|\max _{x \in D_{n}} \bar{z}_{n}(x)-\max _{x \in D_{n}} \bar{z}_{n}^{\prime}(x)\right| \\
& \leq m\left(n^{\eta} \sqrt{2 C m^{2} n^{\eta-1 / 2}}+n^{\eta-1 / 2}\right) .
\end{aligned}
$$

\subsubsection{Independence of the parameters}

A major issue with $D_{n}$ is the condition $x^{K \cap P}+x^{r} \in P$. We would rather have a set of possible values for $x^{r}$ independent of the value of $x^{K \cap P}$. To try to achieve that goal, let

$$
P_{n}=\left\{x \in E^{2}: \forall i \in I, \forall \bullet \in\{X, Y\}, x_{i}^{\bullet} \geq-a_{i}^{\bullet}+2 C m n^{\eta-1 / 2}, \forall i \notin I, x_{i}^{X} \geq 0, x_{i}^{Y} \geq 0\right\} \subset P,
$$

and let $D_{n}^{\prime} \subset D_{n}$ be given by

$$
D_{n}^{\prime}=\left\{\left(x^{K \cap P_{n}}, x^{r}\right) \in\left(K \cap P_{n}\right) \times E^{2}:\left\|x^{r}\right\|_{\infty} \leq 2 C m n^{\eta-1 / 2}, x^{K \cap P_{n}}+x^{r} \in P\right\} .
$$

Now, recalling the definition $E^{\prime}=\left\{x \in E: \forall i \in I^{c}, x_{i} \geq 0\right\} \subset E$, we have that

$$
D_{n}^{\prime}=\left\{\left(x^{K \cap P_{n}}, x^{r}\right) \in\left(K \cap P_{n}\right) \times E^{\prime 2}:\left\|x^{r}\right\|_{\infty} \leq 2 C m n^{\eta-1 / 2}\right\} .
$$

For $\left(x^{K \cap P}, x^{r}\right) \in D_{n}$, and for $n$ large enough so that $\frac{2 C m n^{\eta-1 / 2}}{a_{\min }} \leq 1$, it follows that, letting $x^{\prime K \cap P}:=\left(1-\frac{2 C m n^{\eta-1 / 2}}{a_{\min }}\right) x^{K \cap P},\left(x^{\prime K \cap P}, x^{r}\right) \in D_{n}^{\prime}$, so by (2.7)

$$
\left|\max _{x \in D_{n}^{\prime}} \bar{z}_{n}^{\prime}(x)-\max _{x \in D_{n}} \bar{z}_{n}^{\prime}(x)\right| \leq|I|\left(n^{\eta} \sqrt{\frac{2 C m^{2} n^{\eta-1 / 2}}{a_{\min }}}+n^{\eta-1 / 2}\right) .
$$


On the limiting law of the length of the longest common and increasing subsequences

\subsubsection{Connections with the functions of Lemma 2.2}

Let us now prove that for $n$ large enough,

$$
\max _{x \in D_{n}^{\prime}} \bar{z}_{n}^{\eta}(x)=\max _{\lambda \in a+K \cap P_{n}} \mathfrak{m}\left(v^{X}\left(\lambda^{X}\right), v^{Y}\left(\lambda^{Y}\right)\right) .
$$

Fix $x^{K \cap P_{n}} \in K \cap P_{n}$. Applying the previous lemma to $\nu:=v\left(a+x^{K \cap P_{n}}\right)$, since $\|\nu\|_{\infty} \leq n^{\eta}$, by Lemma 1.5

$$
\begin{aligned}
\max _{\substack{x^{r} \in E^{\prime 2} \\
\left\|x^{r}\right\|_{\infty} \leq 2 C m n^{\eta}}} \sum_{i=1}^{m}\left[\left(p_{i}^{X} x_{i}^{r, X}+\nu_{i}^{X}\right) \wedge\left(p_{i}^{Y} x_{i}^{r, Y}+\nu_{i}^{Y}\right)\right] & =\max _{x^{r} \in E^{\prime 2}} \sum_{i=1}^{m}\left[\left(p_{i}^{X} x_{i}^{r, X}+\nu_{i}^{X}\right)\right. \\
& \left.\wedge\left(p_{i}^{Y} x_{i}^{r, Y}+\nu_{i}^{Y}\right)\right] \\
& =\mathfrak{m}(\nu),
\end{aligned}
$$

and so

$$
\begin{aligned}
\max _{\substack{x^{r} \in E^{\prime 2} \\
\|_{\infty} \leq 2 C m n^{\eta-1 / 2}}} \bar{z}_{n}^{\prime}\left(x^{K \cap P_{n}}, x^{r}\right)= & \max _{\substack{x^{r} \in E^{\prime 2} \\
\left\|x^{r}\right\|_{\infty} \leq 2 C m n^{\eta-1 / 2}}} \sum_{i=1}^{m}\left[\left(\sqrt{n} p_{i}^{X} x_{i}^{r, X}+\nu_{i}^{X}\right) \wedge\left(\sqrt{n} p_{i}^{Y} x_{i}^{r, Y}+\nu_{i}^{Y}\right)\right] \\
= & \max _{\substack{x^{r} \in E^{\prime 2} \\
x^{r} \|_{\infty} \leq 2 C m n^{\eta}}} \sum_{i=1}^{m}\left[\left(p_{i}^{X} x_{i}^{r, X}+\nu_{i}^{X}\right) \wedge\left(p_{i}^{Y} x_{i}^{r, Y}+\nu_{i}^{Y}\right)\right] \\
= & \mathfrak{m}(\nu) .
\end{aligned}
$$

Finally,

$$
\max _{x \in D_{n}^{\prime}} \bar{z}_{n}^{\prime}(x)=\max _{x \in K \cap P_{n}} \max _{\substack{x \in E^{\prime 2} \\\|x\|_{\infty} \leq 2 C m n^{\eta-1 / 2}}} \bar{z}_{n}^{\prime}\left(x^{K \cap P_{n}}, x^{r}\right)=\max _{\lambda \in a+K \cap P_{n}} \mathfrak{m}\left(v^{X}\left(\lambda^{X}\right), v^{Y}\left(\lambda^{Y}\right)\right) .
$$

\subsubsection{End of the proof}

Just as done with (2.19),

$$
\left|\max _{\lambda \in a+K \cap P} \mathfrak{m}(v(\lambda))-\max _{\lambda \in a+K \cap P_{n}} \mathfrak{m}(v(\lambda))\right| \leq|I|\left(n^{\eta} \sqrt{\frac{2 C m^{2} n^{\eta-1 / 2}}{a_{\min }}}+n^{\eta-1 / 2}\right),
$$

and so, using (2.18), (2.19) and (2.20) (recall that $a+K \cap P=K_{\Lambda^{2}}$ ),

$$
\left|\max _{x \in P} z_{n}(a+x)-\max _{\lambda \in K_{\Lambda^{2}}} \mathfrak{m}(v(\lambda))\right| \leq\left(\frac{2|I|}{\sqrt{a_{\min }}}+m\right) \sqrt{2 C m^{2}} n^{\frac{6 \eta-1}{4}}+(2|I|+m) n^{\eta-1 / 2} .
$$

\section{Consistency with previous results and generalizations}

\subsection{Two words with identical distributions}

As stated in the introductory section, Theorem 1.1 and the conjectured Theorem 1.2 are consequences of our main theorem. Indeed, let $X_{k}$ and $Y_{k}(k=1,2, \ldots)$ have the same distribution, then note that

$$
I=\left\{i \in\{1, \ldots, m\}: p_{i}^{X}=p_{\max }\right\},
$$

and so the multiplicity $k^{*}$ of $p_{\max }$ is equal to $|I|$ and we are in Case b1). It is also clear that

$$
K_{\Lambda^{2}}=\left\{\lambda \in \Lambda^{2}: \forall i \notin I, \lambda_{i}^{X}=\lambda_{i}^{Y}=0\right\}^{2} .
$$


On the limiting law of the length of the longest common and increasing subsequences

In this case, Lemma 1.5 simplifies and gives $\mathfrak{m}(\nu)=S^{X} \wedge S^{Y}$, so our theorem states that the limiting distribution of $Z_{n} / \sqrt{p_{\max }\left(1-p_{\max }\right)}$ is

$$
\begin{aligned}
& \max _{\lambda \in K_{\Lambda^{2}}}\left[\left(\sum_{i \in I} B_{i}^{X}\left(\sum_{j=1}^{i} \lambda_{j}^{X}\right)-B_{i}^{X}\left(\sum_{j=1}^{i-1} \lambda_{j}^{X}\right)\right) \wedge\left(\sum_{i \in I} B_{i}^{Y}\left(\sum_{j=1}^{i} \lambda_{j}^{Y}\right)-B_{i}^{Y}\left(\sum_{j=1}^{i-1} \lambda_{j}^{Y}\right)\right)\right] \\
& =\max _{0=t_{0} \leq t_{1} \leq \cdots \leq t_{k^{*}}=1}\left[\left(\sum_{i=1}^{k^{*}}\left(B_{i}^{X}\left(t_{i}\right)-B_{i}^{X}\left(t_{i-1}\right)\right)\right) \wedge\left(\sum_{i=1}^{k^{*}}\left(B_{i}^{Y}\left(t_{i}\right)-B_{i}^{Y}\left(t_{i-1}\right)\right)\right)\right],
\end{aligned}
$$

where $B^{X}$ and $B^{Y}$ are two independent $k^{*}$-dimensional Brownian motions on [0,1] with respective covariance matrix defined in Theorem 2.1. The proof of Corollary 3.3 in [5] shows that, by writing $B^{X}$ and $B^{Y}$ as linear combinations of independent standard Brownian motions, $Z_{n}$ is identical in law to

$$
\begin{aligned}
\max _{0=t_{0} \leq t_{1} \leq \cdots \leq t_{k^{*}}=1} \sqrt{p_{\max }} & {\left[\left(\frac{\sqrt{1-k^{*} p_{\max }}-1}{k^{*}} \sum_{i=1}^{k^{*}} \bar{B}_{i}^{X}(1)+\sum_{i=1}^{k^{*}}\left(\bar{B}_{i}^{X}\left(t_{i}\right)-\bar{B}_{i}^{X}\left(t_{i-1}\right)\right)\right)\right.} \\
& \left.\wedge\left(\frac{\sqrt{1-k^{*} p_{\max }}-1}{k^{*}} \sum_{i=1}^{k^{*}} \bar{B}_{i}^{Y}(1)+\sum_{i=1}^{k^{*}}\left(\bar{B}_{i}^{Y}\left(t_{i}\right)-\bar{B}_{i}^{Y}\left(t_{i-1}\right)\right)\right)\right],
\end{aligned}
$$

where now $\bar{B}^{X}$ and $\bar{B}^{Y}$ are two independent $k^{*}$-dimensional standard Brownian motions on $[0,1]$. Dividing both sides by $\sqrt{p_{\max }}$, one obtains the conjectured Theorem 1.2 which reduces to Theorem 1.1 when $k^{*}=m$.

\subsection{Generalization to any fixed sequence of blocks}

As pointed out by an Associate Editor, and also developed, for binary alphabets, in [8], a longest common increasing subsequence can be viewed as a longest common subsequence where letters are aligned in blocks. (For $L C I_{n}$, a non-void block only aligns a single type of letter and the first block consists of the letter $\alpha(1):=1$, then the second one consists of $\alpha(2):=2$ and so on, up to the last block eventually consisting of the letter $\alpha(m):=m$.) So, more generally, one could investigate the longest common subsequences where letters are aligned in blocks of letters $\alpha(1), \ldots, \alpha(l)$, for any $l \geq m$, and where $\alpha:\{1, \ldots, l\} \rightarrow \mathcal{A}_{m}$ is onto. For any fixed $\alpha$, the length of the longest common subsequences where letters are aligned with blocks $\alpha$ is at most equal to $L C_{n}$, the length of the longest common subsequences, and moreover, $L C_{n}$ is the maximum of these lengths over all the possible block-orders $\alpha$ ( $l$ is not fixed). To pass from the block version to $L C_{n}$, there is, however, a major issue of interversion of limits. In what follows, at first, we merely give for any fixed $\alpha$, the limiting law of the length of the (rescaled) longest common subsequences where letters are aligned in blocks $\alpha(1), \ldots, \alpha(l)$, and then the corresponding limiting laws, when allowing for a fixed numbers of such blocks.

Firstly, defining for any $k \in \mathbb{N}, k \geq 2, \Lambda_{k}:=\left\{\lambda \in\left(\mathbb{R}_{+}\right)^{k}=: \lambda_{1}+\cdots+\lambda_{k}=1\right\}$, we claim that:

$$
\max _{\lambda \in \Lambda_{l}^{2}} \sum_{i=1}^{l}\left[\left(p_{\alpha(i)}^{X} \lambda_{\alpha(i)}^{X}\right) \wedge\left(p_{\alpha(i)}^{Y} \lambda_{\alpha(i)}^{Y}\right)\right]=\max _{\lambda \in \Lambda_{m}^{2}} \sum_{i=1}^{m}\left[\left(p_{i}^{X} \lambda_{i}^{X}\right) \wedge\left(p_{i}^{Y} \lambda_{i}^{Y}\right)\right] .
$$

Indeed to see the validity of this equality, note that above the left-hand side is greater or equal than the right-hand side since $\alpha$ is onto, while it is also less or equal since we can partition $\{1, \ldots, l\}$ via $\alpha^{-1}(\{1\}), \alpha^{-1}(\{2\}), \ldots, \alpha^{-1}(\{m\})$ and use the basic inequality $(a \wedge b)+(c \wedge d) \leq(a+c) \wedge(b+d)$. 
On the limiting law of the length of the longest common and increasing subsequences

Next, to adapt the proof of our main theorem, we need to define the set $U^{\alpha}$, as well as all other quantities which depended on $m$ or $p$, with $l$ instead of $m$ and $p_{\alpha(1)}^{\bullet}, \ldots, p_{\alpha(l)}^{\bullet}$ instead of $p_{1}^{\bullet}, \ldots, p_{m}^{\bullet}$. Note also that, when $l>m$, the quantities $p_{\alpha(1)}^{\bullet}, \ldots, p_{\alpha(l)}^{\bullet}$ do not form a probability mass function (their sum is not equal to one), but all their elements are positive which is enough to have everything well defined.

Formally, for example,

$$
U^{\alpha}:=\left\{u \in \mathbb{R}_{+}^{l}: \frac{u_{1}}{p_{\alpha(1)}^{X}}+\cdots+\frac{u_{l}}{p_{\alpha(l)}^{X}} \leq 1, \frac{u_{1}}{p_{\alpha(1)}^{Y}}+\cdots+\frac{u_{l}}{p_{\alpha(l)}^{Y}} \leq 1\right\},
$$

$\phi^{\alpha}: \mathbb{R}^{l} \rightarrow \mathbb{R}$ is given by

$$
\phi^{\alpha}: u \mapsto u_{1}+\cdots+u_{l},
$$

and $I^{\alpha}$ is now defined to be the set of integers $i \in\{1, \ldots, l\}$ such that there exists $u^{i} \in L_{U^{\alpha}}$ with $u^{i}>0$. Using almost the same proof as the one showing the equality of the two maxima in (3.2), we get $\alpha^{-1}(I)=I^{\alpha}$, where $I$ is defined as before. There is no need to redefine the various cases a), b1), b2) here since they coincide with those previously defined when taking $p_{\alpha(1)}^{\bullet}, \ldots, p_{\alpha(l)}^{\bullet}$ instead of $p_{1}^{\bullet}, \ldots, p_{m}^{\bullet}$. For example, "there exists $u \in U^{\alpha}$ maximizing $\phi^{\alpha}$ over $U^{\alpha}$ such that $\frac{u_{1}}{p_{\alpha(1)}^{X}}+\cdots+\frac{u_{l}}{p_{\alpha(l)}^{X}}=1$ and $\frac{u_{1}}{p_{\alpha(1)}^{Y}}+\cdots+\frac{u_{l}}{p_{\alpha(l)}^{Y}}<1^{\prime \prime}$ is equivalent to Case a) defined in Section 1.3. Finally, the function $\mathfrak{m}$ defined in Lemma 1.5 can be extended naturally to $\left(\mathbb{R}^{l}\right)^{2}$.

Within this generalized setting, the proof of Lemma 2.2 carries over, giving us the following theorem for, $L C_{n}^{\alpha}$, the length of the longest common subsequences with blocks $\alpha(1), \ldots, \alpha(l)$.

Theorem 3.1. Let $B^{X}$ and $B^{Y}$ be two independent $|I|$-dimensional Brownian motions defined on $[0,1]$ with respective covariance matrix $C^{X}$ defined by $C_{i, i}^{X}=1$ and $C_{i, j}^{X}=$ $-\sqrt{\frac{p_{\alpha(i)}^{X} p_{\alpha(j)}^{X}}{\left(1-p_{\alpha(i)}^{X}\right)\left(1-p_{\alpha(j)}^{X}\right)}}$, for $i \neq j$ in $I$, and $C^{Y}$ defined in a similar fashion. For all $\lambda \in K_{\Lambda^{2}}^{\alpha}$ and $i \in I^{\alpha}$, set

$$
\begin{aligned}
& V_{i}^{\alpha, X}\left(\lambda^{X}\right)=\sqrt{p_{\alpha(i)}^{X}\left(1-p_{\alpha(i)}^{X}\right)}\left(B_{\alpha(i)}^{X}\left(\sum_{j=1}^{i} \lambda_{j}^{X}\right)-B_{\alpha(i)}^{X}\left(\sum_{j=1}^{i-1} \lambda_{j}^{X}\right)\right), \\
& V_{i}^{\alpha, Y}\left(\lambda^{Y}\right)=\sqrt{p_{\alpha(i)}^{Y}\left(1-p_{\alpha(i)}^{Y}\right)}\left(B_{\alpha(i)}^{Y}\left(\sum_{j=1}^{i} \lambda_{j}^{Y}\right)-B_{\alpha(i)}^{Y}\left(\sum_{j=1}^{i-1} \lambda_{j}^{Y}\right)\right) .
\end{aligned}
$$

If there exists $u \in L_{U^{\alpha}}$ such that $\frac{u_{1}}{p_{\alpha(1)}^{X}}+\cdots+\frac{u_{l}}{p_{\alpha(l)}^{X}}=1$ and $\frac{u_{1}}{p_{\alpha(1)}^{Y}}+\cdots+\frac{u_{l}}{p_{\alpha(l)}^{Y}}<1$, or equivalently if there exists $u \in L_{U}$ such that $\frac{u_{1}}{p_{1}^{X}}+\cdots+\frac{u_{m}}{p_{1}^{X}}=1$ and $\frac{u_{1}}{p_{1}^{Y}}+\cdots+\frac{u_{m}}{p_{m}^{Y}}<1$ (Case a)), then

$$
\frac{L C_{n}^{\alpha}-n e_{\max }}{\sqrt{n}} \underset{n \rightarrow \infty}{\Longrightarrow} Z^{a}:=\max _{\lambda^{X} \in J^{\alpha}} \sum_{i \in I^{\alpha}} V_{i}^{\alpha, X}\left(\lambda^{X}\right) \text {. }
$$

If for all $u \in L_{U^{\alpha}}, \frac{u_{1}}{p_{\alpha(1)}^{X}}+\cdots+\frac{u_{l}}{p_{\alpha(l)}^{X}}=1$ and $\frac{u_{1}}{p_{\alpha(1)}^{Y}}+\cdots+\frac{u_{l}}{p_{\alpha(l)}^{Y}}=1$, or equivalently if for all $u \in L_{U}, \frac{u_{1}}{p_{1}^{X}}+\cdots+\frac{u_{m}}{p_{1}^{X}}=1$ and $\frac{u_{1}}{p_{1}^{Y}}+\cdots+\frac{u_{m}}{p_{m}^{Y}}=1$ (Case b)), then

$$
\frac{L C_{n}^{\alpha}-n e_{\max }}{\sqrt{n}} \underset{n \rightarrow \infty}{\Longrightarrow} Z^{b}:=\max _{\lambda \in K_{\Lambda^{2}}^{\alpha}} \mathfrak{m}\left(V^{\alpha, X}\left(\lambda^{X}\right), V^{\alpha, Y}\left(\lambda^{Y}\right)\right),
$$

where, again, now $\mathfrak{m}$ is defined on $\left(\mathbb{R}^{l}\right)^{2}$.

For instance, for $m=2$ and in the uniform case, the order $\alpha(1)=2, \alpha(2)=1, \alpha(3)=2$ gives the limiting distribution: 
On the limiting law of the length of the longest common and increasing subsequences

$$
\frac{L C_{n}^{\alpha}-n e_{\max }}{\sqrt{n}} \underset{n \rightarrow \infty}{\Longrightarrow} Z^{b}:=\max _{\substack{\lambda_{1}^{X}+\lambda_{2}^{X}+\lambda_{X}^{X}=1 \\ \lambda_{1}^{Y}+\lambda_{2}^{Y}+\lambda_{3}^{Y}=1}} \mathfrak{m}\left(V^{\alpha, X}\left(\lambda^{X}\right), V^{\alpha, Y}\left(\lambda^{Y}\right)\right)
$$

i.e.,

$$
\begin{array}{r}
\frac{L C_{n}^{\alpha}-n e_{\max }}{\sqrt{n}} \underset{n \rightarrow \infty}{\Longrightarrow} Z^{b}:=\frac{1}{2} \max _{\substack{\lambda_{1}^{X}+\lambda_{2}^{X}+\lambda_{3}^{X}=1 \\
\lambda_{1}^{Y}+\lambda_{2}^{Y}+\lambda_{3}^{Y}=1}} \min _{\bullet \in\{X, Y\}}\left(B_{2}^{\bullet}\left(\lambda_{1}^{\bullet}\right)+B_{1}^{\bullet}\left(\lambda_{1}^{\bullet}+\lambda_{2}^{\bullet}\right)-B_{1}^{\bullet}\left(\lambda_{1}^{\bullet}\right)\right. \\
\left.+B_{2}^{\bullet}(1)-B_{2}^{\bullet}\left(\lambda_{1}^{\bullet}+\lambda_{2}^{\bullet}\right)\right) .
\end{array}
$$

Also note that, sometimes, the limit in the above theorem is simply a normal random variable. Indeed, take $p_{1}^{X}=1 / 3, p_{2}^{X}=2 / 3, p_{1}^{Y}=1 / 4, p_{2}^{Y}=3 / 4$, and $\alpha(1)=1, \alpha(2)=2$, then we are in Case a), $I=\{2\}$ and:

$$
\frac{L C_{n}^{\alpha}-n e_{\max }}{\sqrt{n}} \underset{n \rightarrow \infty}{\Longrightarrow} Z^{a}:=\frac{\sqrt{2}}{3} B_{2}^{X}(1) .
$$

This is also, as one would expect, the limiting distribution of the number of 2's in the first word (which is almost equal to $L C_{n}^{\alpha}$ ). However, if we take $\alpha(1)=2, \alpha(2)=1, \alpha(3)=$ 2 , the limit is more involved.

For $b \in \mathbb{N}$ such that $b \geq m$, let now $F_{m}^{b}$ denote the set of all surjections from $\{1, \ldots, b\}$ to $\{1, \ldots, m\}$, and let $L C_{n}^{(b)}$ be the length of the longest common subsequences with $b \geq m$ blocks, with for each letter at least one block of this letter, and still allowing the blocks to have size zero. This is nothing but the maximum, over all the possible $\alpha \in F_{m}^{b}$, of $L C_{n}^{\alpha}$, so, recalling the discussion preceding the statement of Theorem 3.1, we have:

Theorem 3.2. In Case a),

$$
\frac{L C_{n}^{(b)}-n e_{\max }}{\sqrt{n}} \underset{\overline{n \rightarrow \infty}}{\longrightarrow} Z^{a}:=\max _{\substack{\lambda^{X} \in J^{\alpha} \\ \alpha \in F_{m}^{b}}} \sum_{i \in I^{\alpha}} V_{i}^{\alpha, X}\left(\lambda^{X}\right) .
$$

In Case b),

$$
\frac{L C_{n}^{(b)}-n e_{\max }}{\sqrt{n}} \underset{n \rightarrow \infty}{\longrightarrow} Z^{b}:=\max _{\substack{\lambda \in K_{\Lambda}^{\alpha} \\ \alpha \in F_{m}^{b}}} \mathfrak{m}\left(V^{\alpha, X}\left(\lambda^{X}\right), V^{\alpha, Y}\left(\lambda^{Y}\right)\right) .
$$

Proof. The proof of this theorem follows lines of the proof of our previous main result, considering $p_{\alpha(i)}^{\bullet}$ instead of $p_{i}^{\bullet}$.

Note that $L C_{n}$, the length of the longest common subsequences without any conditions on blocks, corresponds to $L C_{n}^{(n+m)}$ (or to be more precise, $L C_{n}^{(b)}$ for any $b \geq m+n-2$ : this is because when, say, there are only two kind of letters involved in the longest common word, we have to take $m-2$ additional empty blocks to make $\alpha$ onto). Although the above theorem requires a fixed number of blocks, say, $b$, it is nevertheless noteworthy that no matter this fixed number,

$$
\lim _{n \rightarrow+\infty} \frac{\mathbb{E} L C_{n}^{(b)}}{n}=e_{\max }
$$

\subsection{Countably infinite alphabet}

To continue, let us consider, as in [5, Section 4], the generalization to countably infinite alphabets. Let the alphabet be $\mathbb{N}^{*}=\{1,2, \ldots\}$, let $\left(p_{i}^{\prime X}\right)_{i \geq 1}$ and $\left(p_{i}^{\prime Y}\right)_{i \geq 1}$ be two probability mass functions on this alphabet, we are now interested in $L C I_{n}^{\infty}$, the length 
On the limiting law of the length of the longest common and increasing subsequences

of the longest common and increasing subsequences over this countably infinite alphabet. Let

$$
\Lambda^{\infty}=\left\{\lambda \in\left(\mathbb{R}_{+}\right)^{\mathbb{N}^{*}}=[0,+\infty)^{\mathbb{N}^{*}}: \sum_{i=1}^{+\infty} \lambda_{i}=1\right\},
$$

and let

$$
e_{\max }^{\infty}=\sup _{\lambda \in\left(\Lambda^{\infty}\right)^{2}} \sum_{i=1}^{+\infty}\left[\left(p_{i}^{\prime X} \lambda_{i}^{X}\right) \wedge\left(p_{i}^{\prime Y} \lambda_{i}^{Y}\right)\right] .
$$

Let $m \in \mathbb{N}, m \geq 2$ be such that $\sum_{i=m}^{+\infty} p_{i}^{\prime X}<e_{\max }^{\infty}$ and $\sum_{i=m}^{+\infty} p_{i}^{\prime Y}<e_{\max }^{\infty}$. Let us consider the distributions over $\{1, \ldots, m\}$ obtained by replacing all the letters greater or equal to $m$ by $m$, namely, let $p_{i}^{X}=p_{i}^{\prime X}$ for $i<m$ and $p_{m}^{X}:=\sum_{i=m}^{+\infty} p_{i}^{\prime X}$, and let $p_{i}^{Y}, 1 \leq i \leq m$, be defined in a similar fashion. Let now $L C I_{n}$ be the length of the longest increasing subsequences formed by replacing all the letters greater or equal to $m$ by $m$, i.e., the longest common and increasing subsequences on $\{1, \ldots, m\}$ associated with the probability mass functions $p^{\prime X}$ and $p^{\prime Y}$. Next we argue, via a sandwiching argument, that when properly centered and scaled (note that $e_{\max }^{\infty}=e_{\max }$ ), $L C I_{n}^{\infty}$ and $L C I_{n}$ tend to the same limit. Indeed, let $L C I_{n}^{*}$ be the length of the longest common and increasing subsequences not using the letter $m$, i.e., the length of the longest common and increasing subsequences on $\{1, \ldots, m-1\}$ associated with the probability mass functions $p^{X}$ and $p^{\prime Y}$ or, equivalently, $p^{X}$ and $p^{Y}$. Since $m \notin I$ (where $I$ is defined with the distribution $\left(p_{i}^{X}\right)_{1 \leq i \leq m}$ and $\left.\left(p_{i}^{Y}\right)_{1 \leq i \leq m}\right),\left(L C I_{n}^{*}-n e_{\max }\right) / \sqrt{n}$ and $\left(L C I_{n}-n e_{\max }\right) / \sqrt{n}$ converge to the same limiting distribution. But,

$$
\frac{L C I_{n}^{*}-n e_{\max }}{\sqrt{n}} \leq \frac{L C I_{n}^{\infty}-n e_{\max }}{\sqrt{n}} \leq \frac{L C I_{n}-n e_{\max }}{\sqrt{n}},
$$

completing the proof.

From the proofs presented above, the passage from two to three or more sequences is clear: the minimum over two Brownian functionals becomes a minimum over three or more Brownian functionals, and such a passage applies to the cases touched upon above and below.

Throughout the text, the two sequences $\left(X_{k}\right)_{k \geq 1}$ and $\left(Y_{k}\right)_{k \geq 1}$ are assumed to be independent with respective i.i.d. components. In view of [6] or [3], one expects that the i.i.d. assumption could be replaced by a Markovian one or even a hidden Markovian one. Moreover, one further expects that the independence of the two sequences is unnecessary and that a potential dependence structure between the two sequences would carry over to corresponding $2 m$-dimensional Brownian functionals, another case at hand could be the hidden Markov framework. Finally, it should also be of interest (as already done in [2] for uniform letters) to study the ramifications/connections of our results with last passage percolation.

\section{Appendix: proof of Lemma 1.5}

Proof. Define $f_{\nu}: E^{\prime 2} \rightarrow \mathbb{R}$ by $f_{\nu}: x \mapsto \sum_{i=1}^{m}\left[\left(p_{i}^{X} x_{i}^{X}+\nu_{i}^{X}\right) \wedge\left(p_{i}^{Y} x_{i}^{Y}+\nu_{i}^{Y}\right)\right]$. In order to prove that $\mathfrak{m}(\nu)$ is well defined and (1.20), it is enough to prove that for all $x \in E^{\prime 2}$, there exists $x^{\prime} \in E^{\prime 2}$ such that $\left\|x^{\prime}\right\|_{\infty} \leq 2 C m\|\nu\|_{\infty}$ and $f_{\nu}\left(x^{\prime}\right) \geq f_{\nu}(x)$. Let $x \in E^{\prime 2}$. Firstly, assume that $x \in P$ (recalling (2.14)). If $f_{\nu}(x)<f_{\nu}(0)$, taking $x^{\prime}=0$ works, so assume $f_{\nu}(x) \geq f_{\nu}(0)$. By (1.10) (applied twice),

$$
-m\|\nu\|_{\infty} \leq f_{\nu}(0) \leq f_{\nu}(x) \leq m\|\nu\|_{\infty}+f(x)
$$

hence $-f(x) \leq 2 m\|\nu\|_{\infty}$ and, by Lemma 2.3, there exists $x^{K \cap P} \in K \cap P$ and $x^{r} \in E^{2}$ such that $x=x^{K \cap P}+x^{r}$ and $\left\|x^{r}\right\|_{\infty} \leq-C f(x) \leq 2 C m\|\nu\|_{\infty}$. But from the definition 
On the limiting law of the length of the longest common and increasing subsequences

of $K, f_{\nu}\left(x^{K \cap P}+x^{r}\right)=f\left(x^{K \cap P}\right)+f_{\nu}\left(x^{r}\right)$, and by (2.16), $f\left(x^{K \cap P}\right)=0$ so $f_{\nu}(x)=f_{\nu}\left(x^{r}\right)$. Moreover, since $x \in P$ and $x_{i}^{K \cap P, \bullet}=0$ for all $i \in I^{c}, x^{r} \in E^{\prime 2}$.

Now, if we do not assume $x \in P$ anymore, observe that for $\varepsilon>0$ small enough, $\varepsilon x \in P$, so $f_{\varepsilon \nu}\left(x^{\prime}\right) \geq f_{\varepsilon \nu}(\varepsilon x)$ for some $x^{\prime} \in E^{\prime 2}$ such that $\left\|x^{\prime}\right\|_{\infty} \leq 2 C m\|\varepsilon \nu\|_{\infty}$. Finally, dividing by $\varepsilon, f_{\nu}\left((1 / \varepsilon) x^{\prime}\right) \geq f_{\nu}(x)$ where $\left\|(1 / \varepsilon) x^{\prime}\right\|_{\infty} \leq 2 C m\|\nu\|_{\infty}$.

In Case b1), let us begin with the subcase $I=\{1\}$. In this instance, $p_{1}^{X}=p_{1}^{Y}=e_{\max }$, while for all $1<i \leq m, p_{i}^{X}<e_{\max }$ or $p_{i}^{Y}<e_{\max }$ (otherwise $i$ would be in $I$ ). We now show that "the maximum of $f_{\nu}$ is realized with the first letter plus one other letter", more precisely, there exists $x \in E^{\prime 2}$ such that $f_{\nu}(x)=\mathfrak{m}(\nu)$ and $\mid\left\{i \in\{2, \ldots, m\}: x_{i}^{X} \neq\right.$ 0 or $\left.x_{i}^{Y} \neq 0\right\} \mid \leq 1$. Indeed, using the same method than in the proof of Lemma 1.6, keeping in mind $\nu_{2}^{\bullet}=\cdots=\nu_{m}^{\bullet}=0$, one can see that there exists some $x$ maximizing $f_{\nu}$ such that $\left\{i \in\{1, \ldots, m\}: x_{i}^{X} \neq 0\right.$ or $\left.x_{i}^{Y} \neq 0\right\}$ has at most two elements, and they can't both belong to $\{2, \ldots, m\}$ otherwise they would be null (by the definition of $E^{\prime}$ ).

Returning to the proof of the lemma, we have shown that

$$
\max _{x \in E^{\prime 2}} f_{\nu}(x)=\max _{i_{0} \in\{2, \ldots, m\}} \sup _{\substack{x \in E^{\prime 2} \\ \forall i \in\{2, \ldots, m\} \backslash\left\{i_{0}\right\}, x_{i}^{*}=0}} f_{\nu}(x) .
$$

Fixing $i_{0} \in\{2, \ldots, m\}$, we have

$$
\sup _{\substack{x \in E^{\prime 2} \\ \forall i \in\{2, \ldots, m\} \backslash\left\{i_{0}\right\}, x_{i}^{\bullet}=0}} f_{\nu}(x)=\sup _{t^{X}, t^{Y}>0}\left[\left(\nu_{1}^{X}-e_{\max } t^{X}\right) \wedge\left(\nu_{1}^{Y}-e_{\max } t^{Y}\right)+\left(p_{i_{0}}^{X} t^{X}\right) \wedge\left(p_{i_{0}}^{Y} t^{Y}\right)\right] .
$$

It is then easily seen that this last supremum does not change with the additional condition $p_{i_{0}}^{X} t^{X}=p_{i_{0}}^{Y} t^{Y}$. (Indeed, if, for example, $p_{i_{0}}^{X} t^{X}>p_{i_{0}}^{Y} t^{Y}$, reducing $t^{X}$ to transform this strict inequality into equality will only increase the sum of the two minima in the definition of $f_{\nu}$.) Hence,

$$
\begin{aligned}
\sup _{\substack{x \in E^{\prime 2} \\
\forall i \in\{2, \ldots, m\} \backslash\left\{i_{0}\right\}, x_{i}^{\bullet}=0}} f_{\nu}(x) & =\sup _{t^{X}>0}\left[\left(\nu_{1}^{X}-e_{\max } t^{X}+p_{i_{0}}^{X} t^{X}\right) \wedge\left(\nu_{1}^{Y}-e_{\max } \frac{p_{i_{0}}^{X}}{p_{i_{0}}^{Y}} t^{X}+p_{i_{0}}^{X} t^{X}\right)\right] \\
& =\sup _{t^{X}>0}\left[\left(\nu_{1}^{X}+\left(p_{i_{0}}^{X}-e_{\max }\right) t^{X}\right) \wedge\left(\nu_{1}^{Y}+\frac{p_{i_{0}}^{X}}{p_{i_{0}}^{Y}}\left(p_{i_{0}}^{Y}-e_{\max }\right) t^{X}\right)\right] .
\end{aligned}
$$

Since $i_{0} \notin I$, it is impossible for both $p_{i_{0}}^{X}-e_{\max }$ and $p_{i_{0}}^{Y}-e_{\max }$ to be positive, so this last supremum is attained at $t^{X}=0$ (and is equal to $\nu_{1}^{X} \wedge \nu_{1}^{Y}$ ) unless $\nu_{1}^{X}<\nu_{1}^{Y}$ and $p_{i_{0}}^{X}-e_{\max }>0$, or $\nu_{1}^{X}>\nu_{1}^{Y}$ and $p_{i_{0}}^{Y}-e_{\max }>0$, in which case the supremum is attained at $t^{X}=\frac{p_{i_{0}}^{Y}}{e_{\max }} \frac{\nu_{1}^{Y}-\nu_{1}^{X}}{p_{i_{0}}^{X}-p_{i_{0}}^{Y}}$, a value at which the two sides in the above minimum are equal to each other. So if $\nu_{1}^{X}<\nu_{1}^{Y}$ and $p_{i_{0}}^{X}-e_{\max }>0$, or $\nu_{1}^{X}>\nu_{1}^{Y}$ and $p_{i_{0}}^{Y}-e_{\max }>0$, then

$$
\sup _{\substack{\left.x \in E^{\prime 2} \\ \ldots, m\right\} \backslash\left\{i_{0}\right\}, x_{i}^{\bullet}=0}} f_{\nu}(x)=\frac{p_{i_{0}}^{X}\left(e_{\max }-p_{i_{0}}^{Y}\right)}{e_{\max }\left(p_{i_{0}}^{X}-p_{i_{0}}^{Y}\right)} \nu_{1}^{X}+\frac{p_{i_{0}}^{Y}\left(p_{i_{0}}^{X}-e_{\max }\right)}{e_{\max }\left(p_{i_{0}}^{X}-p_{i_{0}}^{Y}\right)} \nu_{1}^{Y} .
$$

Assuming that $\nu_{1}^{X}<\nu_{1}^{Y}$, we see that in this case $\mathfrak{m}\left(\nu^{X}, \nu^{Y}\right)=s_{X} S^{Y}+t_{X} S^{X}$. This remains true if $S^{X}=S^{Y}$ (in this case, $\mathfrak{m}\left(\nu^{X}, \nu^{Y}\right)=S^{X}=S^{Y}$ ), and, similarly, when $S^{Y} \leq S^{X}$. The proof of Case b1) is therefore done when $I=\{1\}$.

Still in Case b1), but without the assumption that $I=\{1\}$, assume, without loss of generality, that $I=\{1, \ldots, k\}, k \geq 2$. Define $\tilde{\nu}$ by $\tilde{\nu}_{1}^{\bullet}=S^{\bullet}$ and $\tilde{\nu}_{i}^{\bullet}=0$, for all $i \geq 2$. Let $x^{0} \in E^{\prime 2}$ be defined by $x^{0, Y}=0, x_{1}^{0, X}=\left(S^{X}-S^{Y}+\nu_{1}^{Y}-\nu_{1}^{X}\right) / e_{\max }, x_{i}^{0, X}=\left(\nu_{i}^{Y}-\nu_{i}^{X}\right) / e_{\max }$, 
On the limiting law of the length of the longest common and increasing subsequences

for all $i \in\{2, \ldots, k\}$, and $x_{i}^{0, \bullet}=0$ for all $i \in\{k+1, \ldots, m\}$. Note that for all $x \in E^{\prime 2}$, $f_{\nu}\left(x+x^{0}\right)=f_{\tilde{\nu}}(x)$, so $\mathfrak{m}(\nu)=\mathfrak{m}(\tilde{\nu})$. Moreover, defining $x^{\prime}$ via $x_{1}^{\prime \bullet}=x_{1}^{\bullet}+\cdots+x_{k}^{\bullet}, x_{i}^{\prime \bullet}=0$, for $i \in\{2, \ldots, k\}$, and $x_{i}^{\prime \bullet}=x_{i}^{\bullet}$ everywhere else, we have $x^{\prime} \in E^{\prime 2}$, and

$$
\begin{array}{r}
\left(e_{\max }\left(x_{1}^{X}+\cdots+x_{k}^{X}\right)+\tilde{\nu}_{1}^{X}\right) \wedge\left(e_{\max }\left(x_{1}^{Y}+\cdots+x_{k}^{Y}\right)+\tilde{\nu}_{1}^{Y}\right) \geq\left(e_{\max } x_{1}^{X}+\tilde{\nu}_{1}^{X}\right) \wedge\left(e_{\max } x_{1}^{Y}+\tilde{\nu}_{1}^{Y}\right) \\
+e_{\max }\left(x_{2}^{X}+\cdots+x_{k}^{X}\right) \wedge\left(x_{2}^{Y}+\cdots+x_{k}^{Y}\right), \quad \\
(3.14) \\
\left(e_{\max }\left(x_{1}^{X}+\cdots+x_{k}^{X}\right)+\tilde{\nu}_{1}^{X}\right) \wedge\left(e_{\max }\left(x_{1}^{Y}+\cdots+x_{k}^{Y}\right)+\tilde{\nu}_{1}^{Y}\right) \geq\left(e_{\max } x_{1}^{X}+\tilde{\nu}_{1}^{X}\right) \wedge\left(e_{\max } x_{1}^{Y}+\tilde{\nu}_{1}^{Y}\right) \\
+e_{\max }\left(x_{2}^{X} \wedge x_{2}^{Y}\right)+\cdots+\left(x_{k}^{X} \wedge x_{k}^{Y}\right) .
\end{array}
$$

Hence, $f_{\tilde{\nu}}\left(x^{\prime}\right) \geq f_{\tilde{\nu}}(x)$, and therefore

$$
\mathfrak{m}(\tilde{\nu})=\max _{\substack{x \in E^{\prime 2} \\ \forall i \in\{2, \ldots, k\}, x_{i}^{\bullet}=0}} f_{\tilde{\nu}}(x)
$$

Now applying the subcase $I=\{1\}$ concludes the proof of Case b1).

In Case b2), again assume without loss of generality that $I=\{1, \ldots, k\}, k \geq 2$. Let $L_{1}=(1,0, \ldots, 0,-1,0, \ldots, 0) \in \mathbb{R}^{2 k}$, having $k-1$ zeros between the two non-zero coordinates, let $L_{2}=(0,1,0, \ldots, 0,-1,0, \ldots, 0)$ (still with $k-1$ zeros between the two nonzero coordinates), and iterate this process up to $L_{k}$. Let also $\widetilde{P^{X}}$ be the concatenation of $P^{X} \in \mathbb{R}^{k}$ with $0 \in \mathbb{R}^{k}$, and let $\widetilde{P^{Y}}$ be the concatenation of $0 \in \mathbb{R}^{k}$ with $P^{Y} \in \mathbb{R}^{k}$. The vectors $L_{1}, \ldots, L_{k}, \widehat{P^{X}}, \widehat{P^{Y}}$ are linearly independent since, as already seen in Lemma 1.4, $P^{X}$ and $P^{Y}$ are linearly independent. Now, let $Q$ be a $2 k \times 2 k$ invertible matrix with first rows $L_{1}, \ldots, L_{k}, \widetilde{P^{X}}, \widetilde{P^{Y}}$ (for example, to form such a matrix $Q$, one could complete the first columns with vectors from the canonical basis), let $\Delta \in \mathbb{R}^{2 k}$ be defined by

$$
\Delta_{i}:= \begin{cases}\nu_{i}^{Y}-\nu_{i}^{X} & \text { if } i \in\{1, \ldots, k\} \\ 0, & \text { if } i \in\{k+1, \ldots, 2 k\}\end{cases}
$$

and let $u \in \mathbb{R}^{2 k}$ be defined by

$$
u_{i}:= \begin{cases}\left(Q^{-1} \Delta\right)_{i} & \text { if } i \in\{1, \ldots, k\} \\ 0, & \text { if } i \in\{k+1, \ldots, 2 k\}\end{cases}
$$

We have $u_{i}^{X}-u_{i}^{Y}=\nu_{i}^{Y}-\nu_{i}^{X}$ (where $u^{X}$ is the vector of the first $k$ coordinates of $u$ and $u^{Y}$ the vector of the last $k$ coordinates of $u$ ) for all $i \in\{1, \ldots, k\}$ : these conditions stem from the rows $L_{1}, \ldots, L_{k}$. Moreover, $u_{1}^{X} / p_{1}^{X}+\cdots+u_{m}^{X} / p_{m}^{X}=u_{1}^{Y} / p_{1}^{Y}+\cdots+u_{m}^{Y} / p_{m}^{Y}=0$ (conditions stemming from the rows $\widetilde{P^{X}}, \widetilde{P^{Y}}$ ). Then, expand $u^{X}$ and $u^{Y}$ to $\mathbb{R}^{m}$ by filling with zeros, so that $u:=\left(u^{X}, u^{Y}\right)$ is now in $\left(\mathbb{R}^{m}\right)^{2}$. Setting, for all $i \in\{1, \ldots, m\}$, $y_{i}^{X}:=u_{i}^{X} / p_{i}^{X}, y_{i}^{Y}:=u_{i}^{Y} / p_{i}^{Y}$, lead to $y \in\left(\mathbb{R}^{m}\right)^{2}$, more precisely $y \in E^{\prime 2}$ such that for all $i \in\{1, \ldots, m\}, p_{i}^{X} y_{i}^{X}+\nu_{i}^{X}=p_{i}^{Y} y_{i}^{Y}+\nu_{i}^{Y}$, with moreover

$$
\sum_{i=1}^{m}\left[\left(p_{i}^{X} y_{i}^{X}+\nu_{i}^{X}\right) \wedge\left(p_{i}^{Y} y_{i}^{Y}+\nu_{i}^{Y}\right)\right]=\sum_{i \in I}\left(\frac{p_{i}^{X} y_{i}^{X}+\nu_{i}^{X}+p_{i}^{Y} y_{i}^{Y}+\nu_{i}^{Y}}{2}\right) .
$$

Setting $U^{X}:=\left(u_{i}^{X}\right)_{i \in I} \in \mathbb{R}^{k}, U^{Y}:=\left(u_{i}^{Y}\right)_{i \in I}, R^{X}:=\left(\nu_{i}^{X}\right)_{i \in I}$ and $R^{Y}:=\left(\nu_{i}^{Y}\right)_{i \in I}$, the above expression becomes

$$
\sum_{i=1}^{m}\left[\left(p_{i}^{X} y_{i}^{X}+\nu_{i}^{X}\right) \wedge\left(p_{i}^{Y} y_{i}^{Y}+\nu_{i}^{Y}\right)\right]=\frac{1}{2}\left(U^{X}+R^{X}+U^{Y}+R^{Y}\right) \cdot(1)_{i \in I} .
$$


On the limiting law of the length of the longest common and increasing subsequences

With the notations of Lemma 1.4,

$$
\begin{aligned}
U^{X} \cdot(1)_{i \in I} & =U^{X} \cdot\left(s P^{X}+t P^{Y}\right) \\
& =U^{X} \cdot t P^{Y} \\
& =\left(U^{X}-U^{Y}\right) \cdot t P^{Y} \\
& =\left(R^{Y}-R^{X}\right) \cdot t P^{Y} .
\end{aligned}
$$

Similarly, $U^{Y} \cdot(1)_{i \in I}=\left(R^{X}-R^{Y}\right) \cdot s P^{X}$. So,

$$
\begin{aligned}
\sum_{i=1}^{m}\left[\left(p_{i}^{X} y_{i}^{X}+\nu_{i}^{X}\right) \wedge\left(p_{i}^{Y} y_{i}^{Y}+\nu_{i}^{Y}\right)\right]= & \frac{1}{2}\left(R^{X}-R^{Y}\right) \cdot\left(s P^{X}-t P^{Y}\right) \\
& +\frac{1}{2}\left(R^{X}+R^{Y}\right) \cdot\left(s P^{X}+t P^{Y}\right) \\
= & R^{X} \cdot s P^{X}+R^{Y} \cdot t P^{Y} \\
= & \sum_{i \in I}\left(\frac{s}{p_{i}^{X}} \nu_{i}^{X}+\frac{t}{p_{i}^{Y}} \nu_{i}^{Y}\right)
\end{aligned}
$$

This shows that

$$
\max _{x \in E^{\prime 2}} \sum_{i=1}^{m}\left[\left(p_{i}^{X} x_{i}^{X}+\nu_{i}^{X}\right) \wedge\left(p_{i}^{Y} x_{i}^{Y}+\nu_{i}^{Y}\right)\right] \geq \sum_{i \in I}\left(s \nu_{i}^{X} / p_{i}^{X}+t \nu_{i}^{Y} / p_{i}^{Y}\right) .
$$

Now let $x \in E^{\prime 2}$,

$$
\begin{aligned}
\sum_{i=1}^{m}\left[\left(p_{i}^{X}\right.\right. & \left.\left.x_{i}^{X}+\nu_{i}^{X}\right) \wedge\left(p_{i}^{Y} x_{i}^{Y}+\nu_{i}^{Y}\right)\right]-\sum_{i \in I}\left(\frac{s}{p_{i}^{X}} \nu_{i}^{X}+\frac{t}{p_{i}^{Y}} \nu_{i}^{Y}\right) \\
= & \sum_{i=1}^{m}\left[\left(p_{i}^{X} x_{i}^{X}+\nu_{i}^{X}\right) \wedge\left(p_{i}^{Y} x_{i}^{Y}+\nu_{i}^{Y}\right)\right]-\sum_{i=1}^{m}\left[\left(p_{i}^{X} y_{i}^{X}+\nu_{i}^{X}\right) \wedge\left(p_{i}^{Y} y_{i}^{Y}+\nu_{i}^{Y}\right)\right] \\
= & \sum_{i=1}^{m}\left[\left(p_{i}^{X}(x-y)_{i}^{X}\right) \wedge\left(p_{i}^{Y}(x-y)_{i}^{Y}\right)\right] \\
& =f(x-y) .
\end{aligned}
$$

We have $x-y \in E^{\prime 2}$ (recall, also, that $y_{i}=0$ for all $i \in I^{c}$ ), so for some $c>0,(x-y) / c \in P$, and then $f((x-y) / c) \leq 0$, so $f(x-y) \leq 0$. Hence $\sum_{i=1}^{m}\left[\left(p_{i}^{X} x_{i}^{X}+\nu_{i}^{X}\right) \wedge\left(p_{i}^{Y} x_{i}^{Y}+\nu_{i}^{Y}\right)\right]-$ $\sum_{i \in I}\left(s \nu_{i}^{X} / p_{i}^{X}+t \nu_{i}^{Y} / p_{i}^{Y}\right) \leq 0$ and, finally,

$$
\max _{x \in E^{\prime 2}} \sum_{i=1}^{m}\left[\left(p_{i}^{X} x_{i}^{X}+\nu_{i}^{X}\right) \wedge\left(p_{i}^{Y} x_{i}^{Y}+\nu_{i}^{Y}\right)\right]=\sum_{i \in I}\left(s \nu_{i}^{X} / p_{i}^{X}+t \nu_{i}^{Y} / p_{i}^{Y}\right) .
$$

\section{References}

[1] F. Benaych-Georges and C. Houdré. GUE minors, maximal Brownian functionals and longest increasing subsequences in random words. Markov Processes. Related Fields 21 (2015), 109-126. MR-3381226

[2] J.-C. Breton and C. Houdré. On the limiting law of the length of the longest common and increasing subsequences in random words. Stochastic Process. Appl. 127 (2017), 1676-1720. MR-3630241

[3] C. Houdré and G. Kerchev. On the rate of convergence for the length of the longest common subsequences in hidden Markov models. J. Appl. Probab. 56 (2019), no. 2, 558-573 MR3986952 
On the limiting law of the length of the longest common and increasing subsequences

[4] C. Houdré, J. Lember and H. Matzinger. On the longest common increasing binary subsequence. C.R. Acad. Sci., Paris Ser. I 343 (2006), 589-594. MR-2269870

[5] C. Houdré and T. J. Litherland. On the longest increasing subsequence for finite and countable alphabets. High Dimensional Probability V: The Luminy Volume (2009), 185-212. MR-2797948

[6] C. Houdré and T. J. Litherland. On the limiting shape of Young diagrams associated with Markov random words. Markov Processes. Related Fields 26 (2020), 779-838.

[7] M. Kiwi, M. Loebl and J. Matoušek. Expected length of the longest common subsequence for large alphabets. Adv. Math. 197 (2005), 480-498. MR-2173842

[8] Y. Zhang Topics on the length of the longest common subsequences with blocks in binary random words. PhD dissertation, Georgia Institute of Technology (2019).

Acknowledgments. We sincerely thank an Associate Editor and a referee for their detailed readings and numerous comments which greatly helped to improve this manuscript. 


\section{Electronic Journal of Probability Electronic Communications in Probability}

\section{Advantages of publishing in EJP-ECP}

- Very high standards

- Free for authors, free for readers

- Quick publication (no backlog)

- Secure publication $\left(\mathrm{LOCKSS}^{1}\right)$

- Easy interface (EJMS²)

\section{Economical model of EJP-ECP}

- Non profit, sponsored by $\mathrm{IMS}^{3}, \mathrm{BS}^{4}$, ProjectEuclid ${ }^{5}$

- Purely electronic

\section{Help keep the journal free and vigorous}

- Donate to the IMS open access fund ${ }^{6}$ (click here to donate!)

- Submit your best articles to EJP-ECP

- Choose EJP-ECP over for-profit journals

\footnotetext{
${ }^{1}$ LOCKSS: Lots of Copies Keep Stuff Safe http://www. lockss.org/

${ }^{2}$ EJMS: Electronic Journal Management System http://www.vtex.lt/en/ejms.html

${ }^{3}$ IMS: Institute of Mathematical Statistics http://www.imstat.org/

${ }^{4}$ BS: Bernoulli Society http://www. bernoulli-society.org/

${ }^{5}$ Project Euclid: https://projecteuclid.org/

${ }^{6}$ IMS Open Access Fund: http://www.imstat.org/publications/open.htm
} 